\title{
An Experimental and Theoretical Investigation of the 3sp(d) Rydberg States of Fenchone by Polarized Laser Resonance-Enhanced-Multiphoton- Ionization and Fourier Transform VUV Absorption Spectroscopy
}

Dhirendra P. Singh ${ }^{a}$, Nelson De Oliveira ${ }^{b}$, Gustavo A. Garcia ${ }^{b}$, Arno Vredenborg ${ }^{c, d}$, Ivan Powis ${ }^{a, ~ *}$

a) Dhirendra P. Singh, Prof. Ivan Powis: School of Chemistry, The University of Nottingham, University Park, Nottingham NG7 2RD, UK

b) Dr. Nelson De Oliveira, Dr. Gustavo A. Garcia: Synchrotron SOLEIL, I'Orme des Merisiers, Saint Aubin BP 48, 91192 Gif sur Yvette Cedex, France.

c) Dr. Arno Vredenborg, Institute for Molecules and Materials, Radboud University Nijmegen, Heyendaalseweg 135, 6525 AJ Nijmegen, The Netherlands

d) Dr. Arno Vredenborg, MassSpecpecD BV, Langenkampweg 101, 7522 LL Enschede, The Netherlands

\section{ORCID IDs}

Nelson de Oliveira : $\underline{0000-0001-7828-7206}$

Gustavo A Garcia : 0000-0003-2915-2553

Ivan Powis: 0000-0002-7941-9079

*Corresponding Author:

Tel: $\quad$ +441159513467

E-mail: $\quad$ ivan.powis@nottingham.ac.uk

ๆ Includes Supplementary Information 


\section{Abstract}

The VUV absorption spectrum of fenchone is re-examined using synchrotron radiation Fourier transform spectrometry, revealing new vibrational structure. Picosecond laser $(2+1)$ resonance enhanced multiphoton ionization (REMPI) spectroscopy complements this, providing an alternative view of the 3spd Rydberg excitation region. These spectra display broadly similar appearance, with minor differences that are largely explained by referring to calculated one- and two-photon electronic excitation cross-sections. Both show good agreement with Franck-Condon simulations of the relevant vibrational structures. Parent ion REMPI ionization yields with both femtosecond and picosecond excitation laser pulses are studied as a function of laser polarization and intensity, the latter providing insight into the relative two-photon excitation and one-photon ionization rates. The experimental circular-linear dichroism observed in the parent ion yields varies strongly between the $3 s$ and $3 p$ Rydberg states, in good overall agreement with the calculated two-photon excitation circular-linear dichroism, while corroborating other evidence that the $3 p_{z}$ sub-state plays no more than a very minor role in the $(2+1)$ REMPI spectrum.

Vibrationally resolved photoelectron spectra are recorded with picosecond pulse duration $(2+1)$ REMPI at selected intermediate vibrational excitations. The $3 s$ intermediate state displays a very strong $\Delta v=0$ propensity on ionization, but the $3 p$ intermediate evidences more complex vibronic dynamics, and we infer some $3 p \rightarrow 3 s$ internal conversion prior to ionization.

\section{Introduction}

Fenchone is a naturally occurring chiral terpenoid compound $\left(\mathrm{C}_{10} \mathrm{H}_{16} \mathrm{O}\right)$ that contributes its distinctive odour to plants such as fennel. Alongside its many claimed uses as an essential oil extract (perfumery, aromatherapy, flavouring, and antimicrobial agent) it is also commonly adopted as a

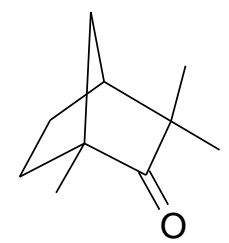

Scheme 1 1R-(-)-fenchone

benchmark molecule with established chiroptical techniques such as electronic absorption circular dichroism (ECD) ${ }^{[1,2]}$, vibrational circular dichroism (VCD) ${ }^{[3]}$, Raman optical activity (ROA) ${ }^{[4]}$, and optical rotatory dispersion (ORD) ${ }^{[5]}$. Importantly in this context, fenchone has a rigid bicyclic structure that prevents it adopting multiple conformations, consequently removing ambiguity about the structural forms present in various media. It also lends itself to theoretical benchmarking ${ }^{[6]}$ and has been particularly of interest in investigations that address solvation effects in CD spectra ${ }^{[2,7,8]}$. These latter studies are further enhanced with fenchone since its relatively high vapour pressure 
facilitates complementary gas phase studies that can thus directly compare solvated and nonsolvated molecular environments ${ }^{[9,10]}$. More recently introduced gas phase chiroptical techniques have continued to choose fenchone as a benchmark. These include cavity ring-down polarimetry ${ }^{[10]}$, and photoelectron circular dichroism (PECD) measured with synchrotron radiation in both soft X-Ray C $1 s$ ionization ${ }^{[11]}$ and vacuum ultraviolet (VUV) valence band ionization ${ }^{[12,13]}$. Even more recently, fenchone has been a primary molecule of choice in fundamental investigations of laser multiphoton ionization PECD ${ }^{[14-20]}$ including pump-probe time-resolved measurements ${ }^{[21,22]}$. Complementing the fundamental laser-based studies, multiphoton ion yield ${ }^{[23]}$ and enantiomeric excess determination $[24,25]$ experiments have addressed more analytical applications using fenchone as a testcase.

However, despite this rather intensive uptake of fenchone in resonance enhanced multiphoton studies of chiral molecules, there remains relatively little background detail concerning its excited state spectroscopy and behaviour. Older measurements of the He I photoelectron spectroscopy of fenchone ${ }^{[26-28]}$ mapped out the valence band ionization profile at low resolution. Very much higher resolution was achieved in resonance enhanced multiphoton ionization (REMPI) spectra obtained using nanosecond pulse lasers for two photon excitation via the $3 s^{[29,30]}$ and, more recently, an extended scan to the include the $3 p$ region ${ }^{[18]}$, but with only limited analysis. Much of the understanding of the $n=3$ Rydberg states that putatively feature as intermediates in studies using REMPI ionization ${ }^{[14-16,18,20-25]}$ is founded upon the VUV absorption spectrum and accompanying calculations reported in 1997 by Pulm et al ${ }^{[2]}$.

In this paper we report a new, wide range (5.7 eV - $9 \mathrm{eV}$ ) absorption spectrum, recorded using VUV Fourier transform (FT) spectrometry, that spans from the onset of the Rydberg excitations in fenchone to above its ionization energy (I.E). This is complemented and directly contrasted with a ps laser $(2+1)$ REMPI spectrum of the $n=3$ Rydberg region recorded at comparable resolution, and the spectroscopy is further elucidated using various TDDFT and ADC calculations to obtain cross-sections and expected vibrational distributions. Laser intensity dependence measurements made with ps and fs pulse duration lasers are recorded to help establish the relative importance of the two-photon absorption, ionization, and relaxation processes in the REMPI spectrum. Polarization dependent measurements of the parent ion yields are then able to be used to estimate the circular-linear dichroism in the two-photon absorption and, by comparison with calculated two-photon crosssection data, shed further light on the electronic state involvement. Finally, vibrationally resolved photoelectron spectra, generated with ps excitation, are recorded across the 3sp Rydberg region and these not only provide new insight into the cation vibrational distribution following the ionization step, but also suggest very different roles for vibrational dynamics in the intermediate Rydberg states. 


\section{Experimental Methods}

\section{VUV Absorption}

The absorption spectrum of fenchone was recorded using the VUV FT spectrometer on the DESIRS beamline at Soleil synchrotron facility. The FT spectroscopy experimental branch has been previously described in detail, here we shall give only the relevant characteristics ${ }^{[1,32]}$. DESIRS is an undulator based beamline that provides the continuum background for the absorption measurement in the shape of a pseudo-gaussian 7\% spectral bandwidth that can be tuned over a large UV-VUV range ${ }^{\text {[33] }}$. Fourteen different spectral windows were required in order to cover the range $5-10 \mathrm{eV}$. A windowless gas cell installed in vacuum inside the multipurpose environmental sample chamber of the FT spectroscopy branch was used for the measurement.

Following three freeze-pump-thaw cycles, room temperature fenchone vapour was admitted to the windowless gas cell via a needle valve to control pressure. Typical entrance pressures cover the range $0.10-0.27$ mbar and were set for each individual spectral window in order to record approximately a $50 \%$ absorbance spectrum.

The FT spectrometer was set to provide a spectral resolution of $17.2 \mathrm{~cm}^{-1}$. In the frame of our experimental conditions, an inspection of the spectrum gave no evidence for any feature requiring a better spectral resolution. During the campaign, and due to technical problems, it was not possible to set the absolute absorption cross-section scale by the procedure usually adopted ${ }^{[32]}$. Nevertheless, the cross-section was calibrated against a limonene spectrum recorded under comparable flowing conditions, itself cross-calibrated against literature values ${ }^{[34]}$.

\section{VUV Photoionization}

Single photon ionization to the $\mathrm{X}^{+}$ground state cation of fenchone was studied using the DELICIOUS 3 double velocity map imaging (VMI) photoelectron-photoion coincidence (PEPICO) spectrometer ${ }^{[35]}$ on the DESIRS beamline at Soleil. Fenchone was contained in a small reservoir, heated to $100 \mathrm{C}$, immediately behind a $70 \mu \mathrm{m}$ nozzle. A seeded molecular beam was created by expansion through the nozzle into vacuum using Argon carrier gas at 3.5 bar pressure. After passing a two-stage skimmer assembly the resulting seeded beam entered the main spectrometer chamber where it intersected the synchrotron beam. By recording electrons and ions in coincidence, any ion fragmentation could be monitored as a function of ion electronic state/internal energy by examining the electron energy tagged ion mass spectra. Conversely, photoelectron measurements could be associated with mass- and translational energy-selected ions (the latter achieved by spatial selection 
at the position sensitive ion detector). Other details of VUV ionization measurements using the DELICIOUS 3 VMI apparatus have been provided previously ${ }^{[13,36]}$.

\section{Multiphoton Spectroscopy}

$(2+1)$ resonance enhanced multiphoton ionization (REMPI) spectra were recorded around $400 \mathrm{~nm}$ using either picosecond or femtosecond duration laser pulses and velocity map imaging (VMI) spectrometers.

The picosecond laser system consists of a Ti:Sapph oscillator (Coherent, Vitesse), a regenerative amplifier (Coherent, Legend Elite), two single pass amplifiers (Coherent) and two optical parametric amplifiers (Light Conversion, TOPAS), although only a single optical branch was used for these measurements. The input beam to the TOPAS system has a fixed wavelength of $800 \mathrm{~nm}$, a pulse width of $1.3 \mathrm{ps}$, a repetition rate of $1 \mathrm{kHz}$ and a power of $3.4 \mathrm{~W}$. The output beam from the TOPAS system has an independently tuned fundamental wavelength range from 1100 to $2950 \mathrm{~nm}$ that is subsequently converted to ultraviolet wavelengths using sum-frequency and higher-harmonic generation. For the present work output wavelengths in the range of 350-450 nm were produced to excite the $(2+1)$ REMPI process. These UV wavelengths were calibrated by a comparison with a number of atomic resonance lines obtained from hollow-cathode lamps. The laser beam is focused into a photoelectron spectrometer chamber (operating pressure of $3 \times 10^{-8} \mathrm{mbar}$ ) using a $30 \mathrm{~cm}$ focal length lens, where it may spatially and temporally overlap a pulsed molecular sample beam.

The energy and angular distributions of the ejected photoelectrons were measured using a VMI photoelectron spectrometer that has been described previously ${ }^{[37]}$. Briefly, emitted photoelectrons were accelerated from a standard VMI instrument source design, travelled $400 \mathrm{~mm}$ along a field-free region, before striking a $40 \mathrm{~mm}$ dual microchannel plate/phosphor screen detector (Photek). The field-free region of the spectrometer was shielded from magnetic fields by a mu-metal tube. The microchannel plate/phosphor screen detector was gated so that it was only activated when the electrons arrived, and a CCD camera (Basler A302f) captured the image on the phosphor screen. The recorded 2D images are inverted to reconstruct the 3D velocity distributions of the photoelectrons using the pBasex method ${ }^{[38]}$ and photoelectron spectra obtained from the radial velocity distributions. The electron energy scale was calibrated by recording the known $\mathrm{S}_{1}$ origin of aniline [39].

A seeded molecular beam of fenchone was generated by bubbling 1 bar He carrier gas through a room temperature reservoir and thence through a $1 \mathrm{kHz}$ pulsed nozzle ${ }^{[40]}$ (Amsterdam Piezo Valve) into an expansion chamber. The beam was passed into the VMI spectrometer chamber via a single 
stage skimmer where it was intersected by the laser beam. Although milder expansion conditions than used for the VUV measurements, these arrangements still provide moderate cooling.

Some further measurements were made with a $3 \mathrm{kHz}, 150 \mathrm{fs}$ pulse duration laser coupled with a dual imaging electron-ion coincidence spectrometer, again as previously described. ${ }^{[41-43]}$

With both laser systems the photon intensities $\left(\mathrm{W} \mathrm{cm}^{-2}\right)$ in the interaction region of the spectrometer are derived from measured incident power using the laser temporal properties and an estimate of the beam focus. For this we assumed gaussian beam optics to arrive at a conservative estimate of $60 \mu \mathrm{m}$ diameter for both lasers (which employed identical focusing arrangements); this focus size estimate probably contributes the greatest uncertainty in our intensities.

\section{Computational Details}

Vertical excitation energies of the excited singlet states of fenchone were performed at the MP2/6$31 \mathrm{G}^{* *}$ optimised ground state neutral geometry using the time-dependent density functional method (TDDFT) with the CAM-B3LYP functional, as implemented in the Gaussian16 $6^{[44]}$ computational package. TDDFT calculations use a single reference ground state and, by using the same exchange-correlation functional for ground and excited state descriptors, incorporate an implicit treatment of differential-correlation effects. Excitation energies and oscillator strength calculations were obtained with d-Aug-cc-pVDZ, d-Aug-cc-pVTZ, and the triple-zeta SNST ${ }^{[45][46]}$ basis sets, and these are summarised in Table 1.

Previous systematic investigations have shown that TDDFT calculations made with range-separated functionals, in particular CAM-B3LYP, can be competitive with more expensive ab initio excited state methods that provide more extensive electron correlation corrections, and in some cases TDDFT can provide almost identical accuracy as do benchmark CCSD calculations in predicting Rydberg excitation energies, for example in comparisons made for acetone ${ }^{[47,48]}$. For comparison and validation in this specific instance of fenchone, a larger ketone, we repeated our TDDFT calculations using the perturbation based $2^{\text {nd }}$ order algebraic diagrammatic construction $(A D C(2))$ method and the EOM-CCSD (equation-of-motion (EOM) formalism with multi-reference CCSD ground state), both as implemented in the Q-Chem package ${ }^{[49]}$.

These methods scale unfavourably (respectively $\mathcal{O}\left(\mathcal{N}^{5}\right)$ and $\mathcal{O}\left(\mathcal{N}^{6}\right)$ ) compared with the approximately quadratic scaling of TDDFT, and so can become prohibitively demanding in a molecule the size of fenchone. We have carefully evaluated an alternative basis set of reduced size, which we here designate as cc-pVDZ+R, for this purpose. This employs an un-augmented cc-pVDZ atomic basis, with a family of $s p d f$ diffuse Rydberg-like functions placed at the molecular centre-of-mass, as 
prescribed by Kauffman ${ }^{[50]}$ and that range up to $n=4.5$ ( $n=4$ for the $f$ functions). In TDDFT calculations cc-pVD(T)Z+R yields nearly identical energies and properties to $d-A u g-c c-p V D(T) Z$ but with a nearly $50 \%$ reduction in the number of basis functions. A comparison made with $\operatorname{ADC}(2)$ with d-Aug-cc-pVDZ similarly finds nearly identical energies and oscillator strengths are returned with the smaller cc-pVDZ+R basis. The requirements of an EOM-CCSD calculation with the d-Aug-cc-pVDZ basis exceeded the available computational resources (memory and storage) and only the cc$p V D Z+R$ calculations are available. However, we have successfully concluded a comparison of these two basis sets with both TDDFT and EOM-CCSD calculations made for a very similar terpene, 3Carene ${ }^{[51]}$; these further confirm the excellent agreement of the Rydberg state energies returned by either basis set. Our fenchone results for $A D C(2)$ and EOM-CCSD methods are presented in Table 2. Also shown in Table 2 are the results of previous CCSD calculations ${ }^{[17]}$, specifically comparing the excited state energies obtained with a d-Aug-cc-pVDZ and Ryd-TZ bases. The latter is similarly limited in size by using un-augmented triple-zeta atomic basis functions plus a single exponent $s, p, d$ additional diffuse function placed on either the $\mathrm{O}$ atom or at the molecular $\mathrm{CM}$. It can be seen that the full d-Aug-cc-pVDZ basis yields excited state energies that diverge significantly as the level of excitation increases, though this becomes less marked with a triply augmented pure atomic basis set [17]. The CCSD/Ryd-TZ and EOM-CCSD/cc-pVDZ+R calculations provide very similar energies (within $\leq 0.05 \mathrm{eV}$ ) for the Rydberg excitations, and as will be seen in the following section provide good agreement with experimental spectra. However, the TDDFT energies (Table 1) are also closely similar, CAM-B3LYP/d-Aug-cc-pVDZ results displaying <0.1 r.m.s difference with our EOM-CCSD results over the ten excited states listed, for a very much reduced computational cost. Such an energy difference is in any case in line with the expected overall accuracy of EOM-CCSD methods ${ }^{[52]}$. With caveats about the selection of basis set we thus mirror the favourable method comparison demonstrated for acetone ${ }^{[47]}$.

While ADC(2) offers slightly improved computational costs compared to CCSD methods, we find here that it provides less plausible excitation energies in comparison with either experiment (see below) or other computational approaches including TDDFT-CAM-B3LYP (see Table 2). The authors of Ref. [17] also compared CC2 (a reduced cost linear-response second-order coupled-cluster method) with CCSD but found it also returned poor energy estimates, typically some $0.7 \mathrm{eV}$ too low, confirming an earlier general conclusion that CC2 performs badly in the calculation of Rydberg states ${ }^{[53]}$.

Two-photon absorption (TPA) tensors, relevant for discussion of the $(2+1)$ REMPI measurements in the $3 s, 3 p$, and low $3 d$ regions, were obtained by using a TDDFT/d-Aug-cc-pVDZ calculation making use of single residue quadratic response theory, as implemented in DALTON ${ }^{[54]}$, by an ADC(2)/d-Aug- 
cc-pVDZ calculation using the Intermediate State matrix Representation ${ }^{[55]}$ (ISR), and by the EOMCCSD response theory, both the latter using $Q-C h e m{ }^{[49]}$.

These two-photon absorption cross-sections, $\sigma^{(2)}$, provided in Table 3 for either linear or circular polarized light, were calculated from the TPA tensors using the formulation of McClain ${ }^{[56,57]}$. Excessive resource demands prevented an EOM-CCSD calculation with the full d-Aug-cc-pVDZ basis, and the results in Table 3 were obtained with the reduced size cc-pVDZ+R basis discussed above. Also in Table 3 we include the comparable CCSD two photon excitation results ${ }^{[17]}$, these also restricted to the reduced Ryd-TZ basis, presumably by similar resource considerations.

Excited states have been classified as $3 s, 3 p$ etc. by examination of their principal contributing orbitals. The identification as Rydberg states is confirmed by the $\left\langle r^{2}\right\rangle$ expectation values, characterising the spatial extents, that are shown compared to those of the neutral ground state in Table 1. To facilitate further discussion the $3 p$ Rydberg states have been identified as $3 p_{\mathrm{x}}, 3 p_{\mathrm{y}}$, and $3 p_{z}$ with the axis designations chosen by reference to the local $C_{2 v}$ (planar) geometry of the carbonyl group, following that convention $p_{x}$ is normal to the plane of the $\mathrm{C}-\mathrm{C}(\mathrm{O})-\mathrm{C}$ grouping, and $p_{z}$ parallel to the $\mathrm{C}=\mathrm{O}$ bond (the $\mathrm{C}_{2}$ symmetry axis). While the $s, p$, or $d$ character is well developed, the nominal $3 p_{\mathrm{x}}$ Rydberg has mixed in a little more valence $\pi^{*}$ (sharing the same $b_{1}$ symmetry in the localised $C_{2 v}$ carbonyl environment) character.

Harmonic vibrational frequencies were calculated with Gaussian $16^{[44]}$ for the neutral and cation ground states using DFT and MP2, and for the low-lying Rydberg states using TDDFT with CAM-B3LYP functional, all with various basis sets. In the latter case geometry optimisations occasionally failed to converge to a global minimum, but the SNST basis set provided reliable results across the Rydberg states of interest. A previous systematic study has shown that the combination of CAM-B3LYP and the SNST basis performs well for the calculation of Rydberg state chiroptical properties and vibrational structure in the chiral molecule methyloxirane ${ }^{[45]}$. Franck-Condon simulations for vibrational structure ${ }^{[44]}$ were then computed using ground state frequencies computed at the same level of theory in the adiabatic hessian model ${ }^{[58]}$. To the extent that simulations using other basis sets (aug-cc-pVDZ, aug-cc-pVTZ) were available for comparison, no significant deviations from the CAM-B3LYP/SNST results was found.

\section{Results}

\section{Single Photon Rydberg Absorption Spectrum}

Figure 1 shows the absorption spectrum recorded with the VUV FT instrument at the DESIRS beamline Soleil. The scan starts above the region of the first, very weak $n-\pi^{*}$ valence excitation but 
then extends beyond the fenchone ionization threshold $(8.4 \mathrm{eV})$. The $17.5 \mathrm{~cm}^{-1}$ resolution of this new recording is around ten times better than the $0.5-2 \mathrm{~nm}$ resolution spectrum reported by Pulm et al. ${ }^{[2]}$, and while still in broad agreement the structure here is more pronounced, especially at lowest energy. Above $7 \mathrm{eV}$, the absolute cross-section is reasonably similar to this earlier spectrum but between 5 and $7 \mathrm{eV}$ the FT measurement shows an absorption cross-section increase of roughly $30 \%$. The indirect calibration procedure might be the reason for this discrepancy, although the absolute cross-sections for other terpenes ( $\alpha$-phellandrene, $\alpha$-pinene) recorded during the same campaign and determined following the same procedure, are much closer to references found in the literature $[59,60]$. The series of sharp peaks appearing above $8 \mathrm{eV}$ in Fig. 1 are readily attributed to the $A^{1} \Pi-X$

${ }^{1} \Sigma$ system in $\mathrm{CO}^{[61]}$, presumably a photolysis product at these shorter wavelengths. We estimate that the $\mathrm{CO}$ fraction is less than $0.5 \%$ of the fenchone column density, and thus the contribution is negligible regarding the reduced absolute cross-section. Henceforward, we will focus attention on the 3 spd region below $7.5 \mathrm{eV}$.

Also shown in Fig. 1 are calculated vertical excitation energies for the 3spd Rydberg states (Table 1). Accurate Rydberg state calculations by TDDFT are known to pose difficulties and the CAM-B3LYP/dAug-cc-pVDZ positions marked in Fig. 1 have been offset to lower energy by $0.2 \mathrm{eV}$ to emphasise that even though the absolute vertical excitation energies calculated would seem to be overestimated by this amount, the relative $s-p-d$ spacings appear meaningful. A shift of this magnitude is also consistent with the presumably more reliable CCSD excitation energies from previous work ${ }^{[17]}$ that we also have listed in Table 1.

\section{Single Photon VUV Ionization}

Before considering the Rydberg states in more detail it will be helpful to outline fresh aspects of the VUV ionization of fenchone. A recent PEPICO investigation of fenchone ${ }^{[13]}$ reported that no fragmentation occurred on ionization to the HOMO band, but that the higher-lying bands were strongly fragmented. Although not included in the published results, extrapolation of the observed ion yield curves suggests the energetic onset for the first fragmentation to $\mathrm{m} / \mathrm{z} 81$ to be $>9.5 \mathrm{eV}$ (see Fig. S1, Supplementary information).

Figure 2 shows the velocity map imaged photoelectron spectrum (VMI-PES) of fenchone recorded with a photon energy $h v=9.22 \mathrm{eV}$, well below this fragmentation threshold. The expansion conditions for this measurement used 3.5 bar of Ar as a backing gas to promote greater cooling than hitherto, but also dimer formation was noted in the ion mass spectrum. However, by recording the VMI-PES in coincidence with translationally cold ( $<40 \mathrm{meV}$ ion $\mathrm{KE}$ ) $\mathrm{m} / \mathrm{z}=152$ parent ions only, (dissociative) ionization of the dimer could be excluded, leaving just the cold monomer PES. 
Previously reported valence photoelectron spectra of fenchone ${ }^{[12,26,27]}$ have not examined the HOMO band in such detail. In this higher resolution, jet-cooled spectrum broad vibrational structure is evident which is well reproduced by a Franck-Condon simulation. From our empirical alignment of the experimental ionization energy scale with the simulation's internal vibrational energy scale the adiabatic ionization energy (vibrational origin) can be estimated as $8.495 \pm 0.01 \mathrm{eV}$, in good agreement with a previous finding of $8.49 \pm 0.06 \mathrm{eV}^{[16]}$.

\section{REMPI Excitation Spectrum}

In Figure 3 we present a (2+1) REMPI spectrum of fenchone, recorded by monitoring parent ion mass, $\mathrm{m} / \mathrm{z} 152$, while scanning with linearly polarized, picosecond laser pulses. The spectrum has been normalized by pulse energy, which ranged around $12 \pm 2 \mu \mathrm{J}$ across the spectrum. Spectral resolution estimates can be obtained from the two-photon convolution of the transform limited 12 $\mathrm{cm}^{-1}$ linewidth of a 1.3 ps pulse, that is $\sim 17 \mathrm{~cm}^{-1}$ - an exact match for the $17.5 \mathrm{~cm}^{-1} \mathrm{FT}$ spectral resolution. In practice, however, the transform limit is a best-case scenario and from recorded laser line spectra we obtain a more conservative estimate of $\sim 30 \mathrm{~cm}^{-1}$ (after deconvoluting the monochromator bandwidth). The 3s origin is easily identified in the Fig. 3 spectra and has been established more precisely by the higher resolution ns laser REMPI experiments as $5.953 \pm 0.001 \mathrm{eV}$ ${ }^{[16,30]}$. Below this energy the room temperature FT spectrum displays a hot-band structure at 5.93 $\mathrm{eV}$, which is also still weakly visible in the moderately cooled ps REMPI recording (Fig. 3).

\section{Intensity and Polarization Dependence of the REMPI Ion Yield}

Across the 3s Rydberg region, up to the first prominent 3p Rydberg peak (6.4 eV excitation energy), the equivalent three-photon energy will be less than the ion fragmentation threshold identified by VUV ionization, and correspondingly no fragmentation was observed in the ps REMPI ion ToF spectra in this range. With at least an order of magnitude more intensity being generated by the femtosecond laser, the amount of fragmentation observed in the fs REMPI mass spectra does, however, increase, although for pulse energies $<25 \mu \mathrm{J}\left(5 \times 10^{12} \mathrm{~W} \mathrm{~cm}^{-2}\right)$ the extent of fragmentation remains around the $10 \%$ level. Corroborating an expectation of minimal fragmentation, the ion ToF mass spectrum recorded for single photon ionization at $h v=10.5 \mathrm{eV}$, an eV above the fragmentation onset, shows a similarly limited amount of fragmentation (see Figs. S2 \& S3, Supplementary information).

These regimes all contrast greatly with the reported nanosecond REMPI mass spectrum, which shows very extensive fragmentation into multiple fragment channels, predominantly those with mass $<40$ amu, even at the 3 s origin ${ }^{[19]}$. This degree of fragmentation into the smallest fragment ions even exceeds that seen in the $70 \mathrm{eV}$ electron impact mass spectrum ${ }^{[62]}$ indicating that, 
energetically, very large numbers of photons must be absorbed. It is long established that in these ns duration pulse conditions competing photon absorption pathways involving ionization of neutral fragments can open up ${ }^{[63]}$, which carries potential implications for the observed photoelectron emission. Figure 4 presents the mass-selected femtosecond laser power dependence data recorded in the middle of the $3 \mathrm{~s}$ region at $407.5 \mathrm{~nm}$ (equivalent two- and three-photon energies of $6.09 \mathrm{eV}$ and $9.13 \mathrm{eV}$ respectively). It can be seen that starting with $33 \mu \mathrm{J}$ pulse energy $\left(8 \times 10^{12} \mathrm{~W} \mathrm{~cm}^{-2}\right)$ the total fragmentation has increased to $\sim 16 \%$, and as the laser intensity is further increased there is correspondingly increased overall fragmentation and a growth in the production of smaller fragments. For the parent ion production specifically, the slope of the $\log _{10}-\log _{10}$ plot shows a second order power dependence, consistent with the expected $(2+1)$ REMPI mechanism when the twophoton excitation is the rate limiting and subsequent ionization is saturated. For successively smaller fragments the power dependence increases in steps of 1 from 3 to 5 , strongly suggesting the resonant photon dependence may be increasing in the sequence 2-3-4-5 as increasingly more energetic fragmentation channels are opened. This is, of course, consistent with the energetic requirement that at least four $407.5 \mathrm{~nm}$ photons are needed to access even the first $\mathrm{m} / \mathrm{z}=81$ dissociation threshold.

These photon intensities are reduced by at least an order of magnitude when using the picosecond laser system and Figure 5 shows two further examples of the laser intensity measurements, now showing solely non-dissociative parent ionization, that were obtained by picosecond excitation in the $3 s$ region. The gradient of these $\log _{10}-\log _{10}$ plots of the parent ion yield, chosen in these examples to lie at the red $(417 \mathrm{~nm})$ and blue $(391 \mathrm{~nm})$ ends of the $3 \mathrm{~s}$ band, have increased to around 3. Figure 6 summarises the complete set of picosecond laser power dependence gradients measured across the $3 s p$ region.

Also presented in these figures are ratios of the ion yields recorded when the light polarization is switched between left circular and linear (abbreviated as $C / L$ ). These $C / L$ ratios are primarily associated with polarization dependent differences in two-photon electronic excitation crosssections and hence, with additional caveats, may provide insight into the Rydberg states accessed as resonant intermediates ${ }^{[64-66]}$. For each wavelength investigated with picosecond laser excitation repeated $\mathrm{C} / \mathrm{L}$ measurements were made using several laser powers in the range 9-15 $\mathrm{mW}$. While at the longest wavelengths the $C / L$ ratios may suggest a very slight power dependence (see for example the $417 \mathrm{~nm}$ data in Fig. 5), elsewhere there is no discernible $C / L$ power dependence. The picosecond excitation power averaged values that are plotted in Fig. 6 are therefore considered to be fully representative of the circular-linear dichroism ratio, C/L, across the REMPI spectrum. As is 
evident in Fig. 6 , there are very significant $C / L$ changes, both within the $3 s$ region and on moving from the $3 s$ to $3 p$ excitation regions.

A somewhat different approach was adopted to investigate circular-linear dichroism with $407.5 \mathrm{~nm}$ femtosecond excitation. Mass-selected ionization rates were recorded while a quarter waveplate was continuously rotated to cycle the laser through circular-elliptical-linear... polarization. The resulting data was fitted to the expected sinusoidal functional form, and the $C / L$ ratio determined from the sine amplitude. An example is shown in the Supplementary Information (Figure. S4). For the parent ion, using a laser power of $200 \mathrm{~mW}\left(\equiv 1.6 \times 10^{13} \mathrm{~W} \mathrm{~cm}^{-2}\right)$ a C/L ratio of $1.36 \pm 0.04$ was obtained. Simultaneously, the measurement using $200 \mathrm{~mW}$ led to a lower value of $\mathrm{C} / \mathrm{L}=1.21 \pm 0.06$ when monitoring instead the principal $\mathrm{m} / \mathrm{z} 81$ fragment ion channel. Using $70 \mathrm{~mW}\left(\equiv 5.5 \times 10^{12} \mathrm{~W} \mathrm{~cm}\right.$ $\left.{ }^{2}\right)$ a $C / L$ value of $1.32 \pm 0.03$ was returned, although the reduced $\mathrm{m} / \mathrm{z} 81$ fragment intensity now precluded measuring its $\mathrm{C} / \mathrm{L}$.

Our rotating waveplate experiments measuring the circular-linear dichroism (e.g. Fig S4) were not expressly designed to measure purely circular dichroism (CD) in the ionization yield, although this is in principle possible since the laser polarization is continuously varied between RCP and LCP. Using a technique specially developed for the purpose, Boesl and co-workers ${ }^{[23]}$ have reported an asymmetry factor of $4.8 \%$ between RCP and LCP absorption in the multiphoton ion yield of gas phase fenchone, although at much shorter wavelength $(295 \mathrm{~nm})$ than here. However, we noted no such CD around $400 \mathrm{~nm}$, but can claim a sensitivity no better than $3 \%$ for our non-optimum CD measurement.

\section{REMPI Photoelectron Spectra}

Photoelectron spectra of fenchone were recorded for various laser wavelengths (marked in Fig. 6) exciting via $3 s$ and $3 p$ resonances at the two-photon level. An overview of the set of these measurements made with RCP polarization is presented in Figure 7. The ionization energies (IE) shown in this figure are calculated by assuming an overall three-photon process and subtracting the measured electron kinetic energies from the three-photon energy. In the $3 s$ resonance region the PES displays a single sharp peak that steadily moves to higher IE with increasing excitation energy. More complex spectra with additional peaks and structure appear with shorter wavelength excitation into the $3 p$ region. This behaviour will be further considered following discussion of the vibrational structure of the excited states of fenchone in the next section. 


\section{Discussion}

\section{REMPI spectra}

There is clearly a very close resemblance between the VUV single-photon absorption spectrum and the two-photon ps-REMPI spectrum in Fig. 3. However, two discrepancies merit further discussion. The first is the relative intensity of the $3 s$ excitation which appears much stronger in the REMPI spectra. Figure 8 compares the calculated oscillator strengths (proportional to integrated crosssection) for the single photon excitation of the 3sp Rydberg states (see also Table 1). In a lower panel are shown calculated two-photon cross-sections for these states. The conversion of calculated twophoton strengths to macroscopic cross-sections for quantitative experimental comparisons can be problematic ${ }^{[67]}$, but for present purposes only relative cross-sections are required. In both panels it can also be seen that there are differences between the adopted quantum chemical models. Nevertheless, the trends are in both cases clear with $3 p$ transitions being relatively less strongly favoured compared to the $3 \mathrm{~s}$ in a two-photon excitation. The different $3 s: 3 p$ ratios in the VUV and REMPI spectra are thus clearly ascribed to the different photon transition mechanisms for the resonant ground-Rydberg excitations.

The calculated cross-sections in Fig. 8 strongly suggest that in the two-photon resonant spectra of fenchone's $3 p$ states the $3 p_{z}$ contribution will be negligible. Conversely, single photon transitions to $3 p_{z}$ are predicted to be the strongest in the VUV spectrum. Comparing the ps REMPI spectrum and the VUV FT spectrum in Fig. 3, one may note a different profile for the first two prominent peaks in the $3 p$ region ( 6.4 and $6.46 \mathrm{eV}$ ). Particularly for the second of these peaks, the VUV absorption spectrum shows evidence of a splitting of around $30 \mathrm{meV}$. After detailed examination we conclude that visible differences between the ps REMPI and VUV spectra could be attributable to a slightly worse resolution in the former, and indeed the much better resolved ns REMPI spectrum (Fig. 3) clearly shows such splittings in the $3 p$ region ${ }^{[16]}$; we cannot then adduce evidence from these comparisons for a different population of the $3 p$ Rydberg components in a one- or two-photon excitation.

In Figure 9 we compare a consistent set of Franck-Condon vibrational simulations (made with the same CAM-B3LYP functional, SNST basis set, assumed temperature, and width function) for the cation and low Rydberg states of fenchone. (The $3 p_{z}$ Rydberg is omitted because it could not be converged to a stable equilibrium geometry required for the frequency analysis.) All show very similar vibrational structure for the first few tenths of an $\mathrm{eV}$ above the origin and, significantly, the characteristic spacings are comparable to the experimental peak splitting noted above. A direct comparison of three of these simulations with the ps REMPI spectrum is provided in Figure 10. 
Following usual practice calculated harmonic frequencies have been scaled, as noted in the figure caption, for comparison with experiment ${ }^{[68]}$. For the $3 s$ simulation we first note an excellent agreement with experiment for the first few tenths $\mathrm{eV}$, and which remains good up to the $3 p$ onset. Figure 11 demonstrates that this same 3 s simulation, but using a reduced peak width parameter to broaden the stick spectrum, also produces excellent agreement with a much higher resolved nanosecond laser REMPI spectrum ${ }^{[29]}$ around the $3 s$ origin, and so is consistent also with the CC2 simulation for the $3 s$ Rydberg state that appears in Ref. [19].

The $3 p_{y}$ simulation likewise provides a good account of the picosecond $3 p$ spectrum (Fig. 10) up to about $6.7 \mathrm{eV}$. However, using the ps simulations (both having the same assumed width of $15 \mathrm{meV}$ ) as a guide it is evident from Fig. 10 that the width of the experimental REMPI peaks to the low energy side of the $3 s$ band is much less than the corresponding $3 p$ REMPI peaks. In a preceding paragraph it was suggested that the experimental resolution in the ps REMPI $3 p$ band might be worse than that of the FT VUV recording, even though for a 1.3 ps duration pulse we previously estimated a roughly commensurate instrumental resolution. Instead, the question is now seen to be why the ps REMPI peak widths in the $3 p$ region appear broadened compared to those of the $3 s$ band?

The authors of Refs. ${ }^{[16,19]}$ have previously noted that the $3 p$ lines in their ns REMPI spectrum were broader than the $3 s$ lines (the latter most probably reflecting rotational profiles). From the experimental linewidths they were thence able to estimate $\tau \geq 0.6$ ps as a lower lifetime limit for the $3 s$ state. Further, from the extra width of the $3 p$ lines they suggested $\tau \geq 80 \mathrm{fs}$ for the $3 p$ state ${ }^{[16]}$. It is implausible to simply transfer these lifetime broadening arguments to the ps pulse duration REMPI spectrum. Rather, we consider the possibility that the observed ps $3 p$ widths reflect the unresolved excitation of both $3 p_{\mathrm{y}}$ and $3 p_{\mathrm{x}}$ states (we discount $3 p_{\mathrm{z}}$ given its much lower predicted two-photon cross-section). The calculated separations of the $3 p$ sub-states (Tables $1 \& 2$ ) are in the range $30-50 \mathrm{meV}$. These indicate the separations that might be applied when two (or three) $3 p$ sub-state simulations were superposed to generate a full $3 p$ region simulation, and do indeed roughly correspond the experimental observed spacings, as suggested in Ref. [19]. But a 30-50 meV spacing also matches the vibrational spacings evident in the Franck-Condon simulations. Given the close similarity of these Rydberg vibrational simulations (Fig. 10), vibrational spacings, and electronic offsets, it proves impossible to arrive at a unique and unambiguous fitting of two or more simulations with the experiment. We can, however, maintain that the observed $3 p$ REMPI broadening is consistent with the presence of two or more unresolved $3 p$ substate spectra. 


\section{Intermediate State Lifetimes}

A second order laser intensity dependence, as observed for the $150 \mathrm{fs}$ pulse duration, $407.5 \mathrm{~nm}$ laser ionization producing parent ion via the $3 s$ resonance (Fig. 4), is usually taken to indicate saturation of the ionization step ${ }^{[69]}$, such that the two-photon intermediate state excitation is rate determining. A simple first order kinetic model ${ }^{[69]}$ that considers competition between the monitored parent $(\mathrm{m} / \mathrm{z}$ 152 ) ionization and relaxation by non-ionizing- , or unobserved ionization-, channels (see Supplementary Info) gives as an approximate result that $1 / \tau \ll \sigma^{(1)} I$, where $\tau$ is the effective relaxation time, $\sigma^{(1)}$ is the single photon ionization cross-section, and I the intensity. Assuming a typical value of $\sim 10 \mathrm{Mb}$ for the ionization cross-section ${ }^{[70,71]}$, similar to camphor ${ }^{[72]}$, and considering a mid-range fs laser intensity of $1.2 \times 10^{13} \mathrm{~W} \mathrm{~cm}^{-2}$ we obtain that $\tau \gg 4 \mathrm{fs}$.

Similarly, a $2^{\text {nd }}$ order intensity dependence is found for the parent ionization channel in the $3 p$ resonant excitation using ps duration laser pulses around $376 \mathrm{~nm}(6.6 \mathrm{eV})$ (Fig. 6). As above, one may estimate, assuming mid-range ps laser power of $10 \mathrm{~mW}\left(\equiv 3.5 \times 10^{11} \mathrm{~W} \mathrm{~cm}^{-2}\right)$ and $\sigma^{(1)} \approx 10 \mathrm{Mb}$, a lower lifetime limit of $\tau \gg 150 \mathrm{fs}$. In contrast Figs. $4 \& 6$ also show that for ps excitation in the $3 \mathrm{~s}$ resonant region the laser intensity dependence for parent ion production exceeds $2^{\text {nd }}$ order and approaches $3^{\text {rd }}$ order. Conventionally, this now indicates that relaxation into non-ionizing channels is becoming competitive with ionization. Since the laser pulse duration is $1.3 \mathrm{ps}$ we may infer that $\tau$ is of similar magnitude. This is, of course quite consistent with the above deduction of a $4 \mathrm{fs}$ lower bound based on the fs laser result, and with the alternative estimate of $\tau_{3 s} \geq 0.6$ ps based upon observed ns laser REMPI linewidths ${ }^{[16,19]}$. It is also fully consistent with a direct lifetime measurement ${ }^{[22]}$ of $\tau_{3 s}=3.3$ ps made by a delayed pump-probe experiment at $201 \mathrm{~nm}$ (single photon resonance) .

\section{Circular-Linear Dichroism}

Polarization studies of two-photon absorptions, made in the liquid phase, are a well-established technique that can help assign symmetry and hence identify electronically excited states ${ }^{[64-66]}$, but are also can have diagnostic value with chiral molecules that lack any symmetry ${ }^{[73,74]}$. The circularlinear dichroism effect (difference between the circular- and linear- polarized light excitation strengths) in two-photon resonant gas phase REMPI studies appears, however, to have been largely neglected after some early interest ${ }^{[75-77]}$. As was first recognised, the signal (ionization yield) in $(2+1)$ REMPI entails not just the two-photon absorption, but a further ionizing photon interaction that may modify the observed polarization dependence, and hence would generally require more advanced theoretical modelling. However, in the limit of $2^{\text {nd }}$ order laser power dependence, the ionization step 
may be assumed to be saturated, allowing the REMPI signal to be interpreted as primarily due to the two-photon absorption ${ }^{[76]}$. Such limiting conditions are nowadays more likely attained with the higher intensities generated by ultrafast lasers, as here, suggesting a renewed interest for twophoton polarization spectroscopy in gas phase may emerge.

Table 3 compares calculated two-photon polarized excitation cross-sections and circular-linear dichroism for the excited states of fenchone. While the cross-sections obtained by different methods show some systematic differences in magnitude, they follow the same trends. In particular, the cross-section ratios from a given calculation, of interest as the $\mathrm{C} / \mathrm{L}$ dichroism, are in each case similar. We already inferred a saturated ionization step in the $3 p$ REMPI from the $2^{\text {nd }}$ order laser intensity dependence (Fig. 6). Hence, the experimental C/L dichroism ratios of $\sim 0.4$ shown in the $3 p$ region in Fig. 6 can be directly compared with the calculations in Table 2, and are in excellent agreement with the QRT/d-Aug-cc-pVDZ C/L values of 0.4 and 0.38 for, respectively, the $3 p_{y}, 3 p_{x}$ states.

Rather different considerations apply when considering the $3 p_{z}$ state. This is predicted to have the lowest excitation energy of the $3 p$ states (Table 1 ), but while the experimental C/L value at $6.4 \mathrm{eV}$, the apparent $3 p$ threshold, shows a very slight increase to 0.55 , this is nowhere near the predicted $3 p_{\mathrm{z}} \mathrm{C} / \mathrm{L}$ value of 1.48 (Table 3). Even if the experimental $\mathrm{C} / \mathrm{L}$ ratios are considered to be mean values, formed of overlapping $3 p$ sub-states, it is clear that $3 p_{z}$ could be no more than a very minor contributor. This of course validates the same conclusion arrived at by noting the relatively very weak two-photon cross-section for $3 p_{z}$ (Fig. 8).

Much larger, though varying, $C / L$ ratios are observed throughout the $3 s$ region below $6.4 \mathrm{eV}$. In the centre of the 3s REMPI band, around $6.1 \mathrm{eV}$ excitation energy, these are very similar to the predicted $3 s$ circular-linear dichroism of 1.48-1.49 (Table 3) but decrease somewhat towards the band extremities. In fact, one may observe in Fig. 6 a seeming anti-correlation between the laser intensity dependence, which drops from 3.1 to a minimum of $\sim 2.4$ in the $3 s$ band centre, and the $C / L$ ratios, which rise from $\sim 1.1$ to a maximum of $\sim 1.5$ in the $3 s$ band centre.

At the $3 s$ band extremities the near $3^{\text {rd }}$ order laser intensity dependence indicates that the total yields depend on one-photon ionization as much as the two-photon excitation - so a bigger deviation from the pure two-photon excitation dichroism predictions in Table 3, with no explicit incorporation of the ionization step, is to be expected. In contrast, near the $\sim 6.2 \mathrm{eV}$ band centre, the reduction towards $2^{\text {nd }}$ order in the laser intensity dependence may perhaps indicate "partial saturation" of the ionization step. A correspondingly reduced reliance on the one-photon ionization 
specifics would then explain the observed closer agreement between the experimental $C / L$ and the predicted pure two-photon excitation C/L values in Table 3.

A similar inference follows from the femtosecond $C / L$ measurements in the same central region of the $3 s$ band $(407.5 \mathrm{~nm})$. Here the parent ion REMPI is unambiguously only two-photon limited (viz. the $2^{\text {nd }}$ order slope in Fig. 4 ) and the measured C/L value of $1.38 \pm 0.09$ is close to the predicted twophoton circular-linear dichroism for $3 s$ excitation. Conversely, the production of $m / z=81$ fragment displays clear $3^{\text {rd }}$ order intensity dependence and energetically must entail a minimum absorption of four photons. In this case the measured $C / L$ is $1.21 \pm 0.06$, significantly reduced from that of the competing parent ion channel and so indicative of different photoexcitation dynamics. Again, one sees a deviation from the predicted $C / L$ ratios for a resonant two-photon absorption when the photon dependence rises above $2^{\text {nd }}$ order and the contributory role of further photon absorption (in the resonant excitation or subsequent ionization/fragmentation of the intermediate) cannot be ignored.

The variation in laser intensity dependence between the two- and three-photon limiting cases that we observe for excitation wavelengths spanning the 3s band (Fig. 6) suggests a delicately balanced interplay between the complex dynamical factors that govern production of the Rydberg state, its evolution and relaxation, and its ionization. An explanation for the observed $3 s$ band centre dip towards $2^{\text {nd }}$ order might, for example, be that the electron K.E. dependence of the $\sigma^{(1)}$ photoionization cross-section and the Franck-Condon factors conspire to produce a higher net ionization rate near the band centre. Clearly more theoretical and experimental work would be required to confirm this somewhat speculative interpretation.

Finally, we note that $C / L$ values measured at the beginning of the $3 d$ region appearing in Fig. 6 are $\sim 0.5$, significantly different from the two-photon predicted $C / L$ of $\sim 1.3$ appearing for the $3 d_{\left(x 2-y_{2}\right)}$ state in Table 3. However, predictions for the remaining $3 d$ states are very basis set dependent, seeming to reflect different degrees of mixing in the calculation. Given, then, the paucity of experimental data currently available we do not for now attempt further interpretation of the $3 d \mathrm{C} / \mathrm{L}$ results.

\section{Photoelectron Spectra}

A $\Delta v=0$ propensity rule in the intermediate state ionizations studied here can be expected owing to these Rydberg states possessing a cationic molecular core, so that vertical ionizing transitions with no change in quantum number(s) are favoured. That the vibrational structure of the Rydberg states parallels that of the actual cation is here further evidenced by the very similar full Franck-Condon simulations (Fig. 9). Fig. 7 provides an overview of the normalised set of REMPI-PES, while in Figure 
12 we examine closely three examples of the REMPI-PES recorded with linear polarization. The first of these was recorded with a two-photon excitation at the $3 s$ origin $(2 \phi=5.95 \mathrm{eV})$. The single peak in this PES spectrum is measured (by fitting with a gaussian function) to have a width of $35 \mathrm{meV}$ and a peak centred at an ionization energy of $8.49 \mathrm{eV}$ - which is just the adiabatic I.E. we already inferred. This can be assigned, unambiguously, as the $0-0$ transition from a vibrationless $3 s$ Rydberg to the vibrationless ground state cation, although at this laser wavelength the three-photon equivalent energy $(3 \phi=8.93 \mathrm{eV})$ is energetically sufficient to ionize several vibrationally excited states.

The second REMPI-PES shown in Fig. 12 was recorded at a wavelength for which $2 \phi=6.23 \mathrm{eV}$, so proceeding via a vibrational level of the $3 s$ Rydberg state lying well above the origin of the REMPI spectrum. Again, the PES displays a single sharp peak with an I.E. of $8.76 \mathrm{eV}$ and width of $54 \mathrm{meV}$. All the additional two-photon excitation energy $(6.23-5.95=0.28 \mathrm{eV})$ effectively is transferred into the increased peak ionization energy $(8.76-8.49=0.27 \mathrm{eV})$. Once again there is no evidence of ionization into either higher or lower vibrational levels than that of the principal peak. In fact such behaviour, very strongly following the $\Delta v=0$ propensity rule, is clearly evident throughout the $3 \mathrm{~s}$ excitation and gives rise to a distinct "ridge" appearing in the overview PES plots (Fig. 7).

Once the excitation energy $2 \phi \geq 6.4$ is reached, a second ridge, albeit broader, appears in the REMPI-PES overview (Fig. 7) running parallel to the first. This is readily understood as being formed by $\Delta v=0$ excitations in ionization from the $3 p$ state(s). Back-extrapolating, the line of this ridge intersects the line of the adiabatic I.E. at a point $2 \phi=6.4 \mathrm{eV}$, which value we have already identified as being indicative of the $3 p$ thresholds. Similar $3 s$ and (weak) $3 p$ ridges are observed in the ns laser REMPI PES although these measurements extend to only just above the $3 p$ threshold region ${ }^{[19]}$. In the present results, it can be seen that the initial ridge associated with the $\Delta v=0$ ionization of the $3 s$ Rydberg state apparently continues throughout the $3 p$ excitation band. One might first suppose that this is because above $2 \phi=6.4 \mathrm{eV}$ both the $3 s$ and $3 p$ Rydberg states are energetically accessible and so ionization of either can occur in parallel. However, at shorter wavelengths the ionization energies reached along this ridge exceed $9.1 \mathrm{eV}$ and so fall outside the Franck-Condon window for direct vertical ionization from ground state neutral to the cation (Fig. 2). More tellingly, these resonance enhanced ionizations also fall beyond the Franck-Condon envelope for the neutral to 3s Rydberg excitation, as evidenced in Fig. 10. The probability of there being a direct vertical transition from the ground state neutral to the cation via the $3 \mathrm{~s}$ intermediate accounting for the continuation of this " $3 s$ " ridge is therefore quite negligible.

In fact, following the intensity profile along the peak of this " $3 s$ " ridge one sees it essentially first follows the $3 s$ region FC profile seen in Fig. 10. Then, having started to diminish, at the $3 p$ threshold 
the intensity revives and the profile (rather than the absolute intensity) repeats, again following one of the Franck-Condon envelopes (e.g. the $3 p$ ). Recognising that the width of " $3 s$ " ridges has at the same time increased, and the additional contribution made by the broad $3 p$ ridge, it becomes clear that the total electron yield increases sharply, as does the REMPI intensity (Figs. 6, 10) above the 6.4 $\mathrm{eV} 3 p$ threshold. The $2^{\text {nd }}$ order laser intensity dependence recorded for excitation above $6.4 \mathrm{eV}$ indicates that the ionization step is saturated, so that photoelectron intensity would be expected to follow the vibronic cross-section for the intermediate state excitation. Hence we are led to infer that in the region $2 \phi \geq 6.4 \mathrm{eV}$ the strongly FC favoured $3 p$ excitation rapidly dominates over any disfavoured $3 s$ excitation, but that some $3 p$ population can rapidly interconvert to high vibrational levels of the $3 s$ state. It is these highly excited $3 s$ states that are then ionized in $\Delta v=0$ transitions which now will terminate in equally high vibration levels of the cation lying outside the ground state FC window.

Closer examinations of the example REMPI-PES recorded at $2 \phi=6.60 \mathrm{eV}, 0.2 \mathrm{eV}$ above the $3 p$ threshold, (Fig. 12), sheds further light on this behaviour. The first prominent peak in the spectrum, at I.E. $=8.70 \mathrm{eV}$ sits $\sim 0.2 \mathrm{eV}$ above the adiabatic ionization threshold. Hence all excess internal energy above the $3 p$ Rydberg threshold appears as internal energy of the cation. This, and the trends exhibited by adjacent spectra, confirms this peak as a $3 p \Delta v=0$ ionization. However, it is also immediately obvious from the broader width, tailing to higher I.E., that the $\Delta v=0$ propensity is much weakened. The second prominent peak at $9.17 \mathrm{eV}$, identified as a member of the $3 s \Delta v=0$ series, is more intense than the $8.70 \mathrm{eV} 3 p \Delta v=0$ ionization, quite contrary to the relative $3 s, 3 p$ cross-sections at this two-photon excitation energy. It also is much broadened, revealing a substantial weakening of the $\Delta v=0$ propensity which was so rigorously followed in the pure $3 \mathrm{~s}$ excitation region. This weakened propensity supports the contention of very different internal dynamics following excitation above the $3 p$ threshold.

In this REMPI-PES recorded above the $3 p$ intermediate threshold we have marked two apparent vibrational progressions built upon the $\Delta v=0$ transitions (Fig. 12). It is clear that in terms of both energy and intensity these are discontinuous - that is separate and distinct progressions. The empirically identified transitions have been fitted to series with successive spacings reducing slightly, characteristic of weak anharmonicity, and a fundamental spacing of $\sim 0.089 \mathrm{eV}\left(\sim 720 \mathrm{~cm}^{-1}\right)$. While the FC simulations (Fig. 10) do all show a small peak at this energy, it is a composite of several individual transitions, so that the nature of this mode is not readily established. Nevertheless, contrasting this array of final cation vibrational levels with the single vibrational peak developed in 
the $3 s$ resonant photoionization, one sees the evidence for much more complex vibronic dynamics following excitation into the $3 p$ Rydberg intermediate state.

\section{Conclusions}

By recording the FT VUV absorption spectrum of the Rydberg region of Fenchone we have revealed new vibrational structure in the lower energy $3 s p d$ region. Complementing this with a $(2+1)$ REMPI spectrum recorded with a picosecond laser, the electronic state assignments guided by older calculations have been newly corroborated by TDDFT and EOM-CCSD calculations. These calculations further provided a harmonic vibrational analysis of the $3 s$ and $3 p$ Rydberg states which has permitted good Franck-Condon simulations of the vibrational band profiles of both VUV and REMPI spectra that further aid understanding of the spectroscopy. While these are very similar to one another, the greater prominence of the $3 s$ band in REMPI can be explained by reference to calculated one- and two-photon excitation cross-sections.

Extensive use has been made of laser intensity dependence measurements that shed light on the extent of competition between the two-photon excitation, ionization, and relaxation processes in the REMPI spectrum. In particular, the $3 p$ excitations display a second order power dependence from which we deduce that the two-photon absorption is rate limiting. The $3 s$ excitation is similarly second order at higher intensities reached with a femtosecond laser pulse, but as the laser intensity is decreased there are indications that a more subtle interplay of the excitation and relaxation/ionization dynamical processes becomes more controlling, and the observed power dependence becomes intermediate between $2^{\text {nd }}$ and $3^{\text {rd }}$ order.

The investigation of REMPI circular-linear dichroism, the ratio of signal (ion yield) recorded with circular and linear light polarization is also shown to have the capability to aid diagnosis of the spectroscopy. Circular-linear dichroism is well established in two-photon liquid phase spectroscopy, but seems to have been largely overlooked in gas phase REMPI, due to the possibility of ill-defined contributions from any photon dynamics, including ionization, occurring after the two-photon absorption, but nevertheless contributing to the observed effects. Here, we show that the laser power dependence measurements serve as a guide, and when a $2^{\text {nd }}$ order dependence applies the experimental circular-linear dichroism measurements may be in very good accord with theoretical predictions utilising calculated two-photon absorption cross-sections. Conversely, it has been possible to infer from the apparent lack of such agreement that the $3 p_{z}$ sub-state is a vanishingly small contributor to the overall REMPI $3 p$ excitation, corroborating the same inference based upon the relative weakness of the two-photon $3 p_{z}$ cross-section. 
This latter conclusion seems also to be borne out by small differences noted in the vibrational structure of the single-photon VUV and two-photon REMPI $3 p$ spectral regions, although a satisfactory synthesis of the detail using calculated energetics, cross-sections and Franck-Condon factors has so far eluded us. It may be noted that this understanding of the $3 p$ spectrum - arrived at with the aid of laser power and polarization dependent measurements, calculated two-photon transition strengths, and vibrational simulations - differs from a recent tentative assignment that instead hypothesised commensurate contributions made by all three potential $3 p$ sub-states, equally separated by $\sim 0.06 \mathrm{eV}{ }^{[19]}$. In contrast the $3 p_{\mathrm{x}}$ sub-state, here indicated by CCSD calculations (Fig. 8) to have the strongest two-photon excitation, has been suggested as the dominant $3 p$ REMPI contribution on the basis of very different arguments, that compared theoretical and experimental photoelectron circular dichroism values ${ }^{[17]}$.

The REMPI circular-linear dichroism measurements are particularly revealing in the $3 \mathrm{~s}$ excitation, since they appear to be inversely correlated to the photon intensity dependence. As noted above, in regions that clearly tend towards $2^{\text {nd }}$ order intensity dependence, indicating that the two-photon excitation is the rate limiting step, the dichroism tends towards an upper limit derived from the calculated two-photon absorption tensor for the 3 s state. Conversely, when in the weaker red and blue wings of the $3 s$ absorption band the REMPI signal shifts towards observed $3^{\text {rd }}$ order intensity dependence the observed dichroism reduces, presumably reflecting the increased importance of the third ionization photon in the overall dynamics. The evident suppression of this overall three-photon dependence in the band centre must be attributed to a combination of an ionization cross-section, that initially increases from threshold, and Franck-Condon factors for the ionization. Both are in principle able to ensure faster, hence more readily saturated, ionization at the REMPI band peak. Nevertheless, even in regions approaching $3^{\text {rd }}$ order power dependence the intermediate role of the two-photon resonant $3 s$ state continues to be demonstrated by the very strong $\Delta v=0$ propensity for vibrational excitation observed in REMPI-PES across the band.

Helping to validate such interpretation of observed deviations from the calculated two-photon circular-linear dichroism we were able to show using femtosecond excitation pulses, that with these higher laser intensities the parent ion production occurs in competition with formation to a mass 81 fragment ion. The former is unambiguously two-photon limited $\left(2^{\text {nd }}\right.$ order power dependence); the latter process clearly displays a $3^{\text {rd }}$ order power dependence, and energetically requires a minimum of 4 photon absorption, hence is likely a three-photon resonant process. There is then little surprise that a simultaneous mass-selected measurement of the parent and fragment dichroism shows good agreement with the two-photon calculated dichroism, but far poorer agreement for the $3^{\text {rd }}$ order fragment REMPI. 
A series of REMPI photoelectron spectra, recorded at excitation energies throughout the $3 s$ and $3 p$ excitation region, provide much more insight into the vibrational dynamics of these Rydberg states and the cation. Below the $3 p$ excitation threshold, the $3 s$ REMPI-PES show that the anticipated $\Delta v=0$ propensity is rigorously followed, with a single photoelectron peak. Above the $3 p$ threshold a second series of $\Delta v=0$ peaks appear in the REMPI-PES that are clearly attributable to excitation via the $3 p$ Rydberg intermediate, while the $3 s \Delta v=0$ appears to be continued. However, this leads to excitations that clearly fall outside the Franck-Condon envelope reachable from the ground state geometry, and instead have an intensity distribution that tracks the Franck-Condon envelope of the $3 p$ resonant intermediate. From this it is inferred that rather than the $3 s$ and $3 p$ intermediate states being excited in parallel, the dominant mechanism above the $3 p$ threshold is for its excitation to be followed by a rapid internal conversion to highly excited vibrational levels of the $3 s$ state before it is now ionized. A similar conclusion has been drawn from noting the reduced $3 \mathrm{p}: 3$ s ionization ratio in ns REMPI, as compared to fs REMPI, which was taken to indicate $3 p-3$ s internal conversion occurring on some intermediate experimental timescale ${ }^{[19]}$. A further indication here of much more complex vibronic dynamics above the $3 p$ threshold is that the propensity for $\Delta v=0$ ionizations is lessened, with both the principal REMPI-PES peaks showing vibrational progressions ranging to higher vibrational quantum number.

The deployment and interpretation of a number of spectroscopic techniques (VUV-FT absorption, REMPI, REMPI-PES, i²PEPICO) described in this paper has been enhanced by a careful, complementary investigation of the laser power and polarization dependence of the REMPI signals. Circular-linear dichroism appears to have been largely neglected for gas-phase REMPI studies but, as shown here, the nowadays much more widespread availability of ultrafast laser systems provides access to a much greater span of photon intensities which, when coupled with modern techniques for the straightforward computation of two-photon cross-sections, can be exploited to gain more insight into a relatively large, non-symmetric molecular system.

\section{Acknowledgements}

This research was undertaken as part of the ASPIRE Innovative Training Network, which has received funding from the European Union's Horizon 2020 research and innovation programme under the Marie Sklodowska-Curie Grant Agreement No. 674960. DPS acknowledges an ESR fellowship provided by ASPIRE.

Provision of beamtime by Synchrotron Soleil (beamtime Proposal No. 20190535) is gratefully acknowledged and we thank the technical staff at Soleil for their support and for the smooth operation of the facility. 
IP and DPS acknowledge support from LaserLab-Europe (grant agreement 654148, EU H2020 research and innovation programme) award \#LLAMS002376 for access to the Nijmegen fs laser facility.

We are grateful for access to the University of Nottingham High Performance Computing Facility in support of the computational effort.

The support, helpful discussions, and provision of ps laser system by Katharine L. Reid is gratefully acknowledged. Barratt Park and Tom Baer are thanked for providing, in digital form, their ns REMPI spectra of fenchone that appeared in, respectively, Ref [16] and Ref [29]. We thank James O. F. Thompson for helpful discussions and Lipsa Nag for assistance with recording the Soleil VMI-PES.

\section{Keywords}

Circular-Linear Dichroism; Fenchone; Molecular Rydberg State Spectroscopy; Multiphoton Ionization; Photoelectron Spectroscopy 


\section{Table 1}

Vertical Excitation Energies, $E_{v}(e V)$, Oscillator Strengths, $f$, and Spatial Extents relative to the Ground State, $\Delta\left\langle r^{2}\right\rangle$ (a.u.), for Excited States of Fenchone obtained by TDDFT calculations with CAM-B3LYP functional

\begin{tabular}{|c|c|c|c|c|c|c|c|c|c|c|}
\hline \multirow{2}{*}{\multicolumn{2}{|c|}{$\begin{array}{l}\text { BASIS } \\
\text { STATE }\end{array}$}} & \multicolumn{3}{|c|}{ d-Aug-cc-pVTZ } & \multicolumn{3}{|c|}{ d-Aug-cc-pVDZ } & \multicolumn{3}{|c|}{ SNST } \\
\hline & & & $f \times 10^{2}$ & $\Delta\left\langle r^{2}\right\rangle$ & \multirow{2}{*}{$\begin{array}{l}\text { Ev } \\
4.24\end{array}$} & \multicolumn{2}{|c|}{$f \times 10^{2} \quad \Delta\left\langle r^{2}\right\rangle$} & \multirow{2}{*}{$\begin{array}{l}E_{v} \\
4.26\end{array}$} & & \multirow{2}{*}{$\frac{\Delta\left\langle\mathbf{r}^{2}\right\rangle}{-0.8}$} \\
\hline 1 & $\pi^{*}$ & 4.26 & 0.00 & -0.8 & & 0.00 & -0.8 & & 0.00 & \\
\hline 2 & $3 s$ & 6.29 & 0.04 & 51.7 & 6.26 & 0.04 & 51.7 & 6.36 & 0.04 & 39.6 \\
\hline 3 & $3 p_{z}\left(a_{1}\right)^{(a)}$ & 6.72 & 2.41 & 73.6 & 6.69 & 2.42 & 73.5 & 6.82 & 2.91 & 50.6 \\
\hline 4 & $3 p_{y}\left(b_{2}\right)^{(a)}$ & 6.75 & 1.46 & 83.2 & 6.72 & 1.44 & 82.9 & 6.86 & 1.81 & 56.7 \\
\hline 5 & $3 p_{x}\left(b_{1}\right)^{(a)(b)}$ & 6.80 & 0.38 & 83.0 & 6.76 & 0.40 & 83.0 & 6.92 & 0.44 & 53.9 \\
\hline 6 & $3 d_{(x 2-y 2)}$ & 7.19 & 0.57 & 106.1 & 7.16 & 0.55 & 103.6 & 7.38 & 1.08 & 51.6 \\
\hline 7 & $3 \mathrm{~d}$ & 7.28 & 0.63 & 133.3 & 7.25 & 0.66 & 129.3 & 7.48 & 1.25 & 11.2 \\
\hline 8 & $3 d$ & 7.30 & 0.08 & 131.2 & 7.27 & 0.06 & 126.3 & 7.53 & 0.25 & 48.0 \\
\hline 9 & $3 d$ & 7.32 & 1.64 & 144.1 & 7.29 & 1.66 & 138.5 & 7.55 & 0.51 & 57.5 \\
\hline 10 & $3 d$ & 7.34 & 0.14 & 148.9 & 7.31 & 0.17 & 143.0 & 7.58 & 3.64 & 67.4 \\
\hline
\end{tabular}

${ }^{a}$ symmetry and directions indicated for $3 p$ states are relative to the localised planar $\left(\mathrm{C}_{2 v}\right) \mathrm{C}-\mathrm{CO}-\mathrm{C}$ grouping

${ }^{\mathrm{b}}$ the $3 p_{\mathrm{x}}$ orbital has some $\mathrm{CO} \pi^{*}$ character mixed in 


\section{Table 2}

Vertical Excitation Energies, $E_{v}(e V)$, Oscillator Strengths, $f$, and Spatial Extents relative to the Ground State, $\Delta\left\langle r^{2}\right\rangle($ a.u. $)$, for Excited States of Fenchone calculated by $\operatorname{ADC}(2)$ and CCSD methods

\begin{tabular}{|c|c|c|c|c|c|c|c|c|c|c|c|c|}
\hline \multirow{3}{*}{\multicolumn{2}{|c|}{$\begin{array}{l}\text { METHOD } \\
\text { BASIS } \\
\text { STATE (b) }\end{array}$}} & \multicolumn{6}{|c|}{$\operatorname{ADC}(2)$} & \multirow{2}{*}{\multicolumn{3}{|c|}{$\begin{array}{l}\text { EOM-CCSD } \\
\text { cc-pVDZ + R }\end{array}$}} & \multicolumn{2}{|c|}{ CCSD (a) } \\
\hline & & \multicolumn{3}{|c|}{ d-Aug-cc-pVDZ } & \multicolumn{3}{|c|}{$c c-p V D Z$ + R } & & & & \multirow{2}{*}{$\begin{array}{l}\text { Ryd-TZ } \\
E_{v}\end{array}$} & \multirow{2}{*}{$\begin{array}{l}\text { d-Aug-cc-pVDZ } \\
E_{v}\end{array}$} \\
\hline & & $E_{v}$ & $f \times 10^{2}$ & $\Delta\left\langle r^{2}\right\rangle$ & $E_{v}$ & $f \times 10^{2}$ & $\Delta\left\langle r^{2}\right\rangle$ & & $f \times 10^{2(c)}$ & $\Delta\left\langle r^{2}\right\rangle$ & & \\
\hline 1 & $\pi^{*}$ & 4.02 & 4.02 & -0.3 & 4.00 & 0.00 & -0.5 & 4.28 & 0.00 & -0.8 & 4.44 & 4.35 \\
\hline 2 & $3 \mathrm{~s}$ & 5.38 & 5.38 & 74.3 & 5.41 & 0.18 & 77.7 & 6.21 & 0.05 & 64.5 & 6.19 & 6.39 \\
\hline 3 & $3 p_{z}\left(a_{1}\right)$ & 5.70 & 5.70 & 89.6 & 5.72 & 1.09 & 92.6 & 6.56 & 1.98 & 83.8 & 6.53 & 6.85 \\
\hline 4 & $3 p_{y}\left(b_{2}\right)$ & 5.75 & 5.75 & 105.4 & 5.77 & 0.80 & 109.4 & 6.64 & 0.74 & 94.2 & 6.60 & 6.97 \\
\hline 5 & $3 p_{x}\left(b_{1}\right)$ & 5.80 & 5.80 & 106.8 & 5.81 & 0.24 & 110.6 & 6.66 & 0.88 & 98.7 & 6.62 & 7.10 \\
\hline 6 & $3 d$ & 6.18 & 6.18 & 140.4 & 6.20 & 0.34 & 150.4 & 7.08 & 0.26 & 124.5 & 7.04 & 8.00 \\
\hline 7 & $3 d$ & 6.24 & 6.24 & 152.4 & 6.25 & 0.03 & 162.6 & 7.14 & 0.08 & 135.8 & 7.09 & 8.31 \\
\hline 8 & $3 d$ & 6.25 & 6.25 & 163.3 & 6.26 & 0.25 & 174.9 & 7.15 & & 149.1 & 7.10 & 8.59 \\
\hline 9 & $3 d$ & 6.27 & 6.27 & 168.6 & 6.28 & 0.63 & 180.9 & 7.17 & & 155.3 & 7.12 & 8.74 \\
\hline 10 & $3 d$ & 6.28 & 6.28 & 173.2 & 6.29 & 0.29 & 185.0 & 7.20 & & 160.9 & 7.14 & 8.76 \\
\hline
\end{tabular}

${ }^{a} \operatorname{Ref}[17]$

${ }^{\mathrm{b}}$ symmetry and directions indicated for $3 \mathrm{p}$ states are relative to the localised planar $\left(\mathrm{C}_{2 \mathrm{v}}\right) \mathrm{C}-\mathrm{CO}-\mathrm{C}$ grouping

${ }^{c}$ Values for states $8-10$ are unavailable as the required calculations exceeded the available computational resources 


\section{Table 3}

Two-photon cross-sections, $\sigma^{(2)}$, for linear and circular polarized excitation (in atomic units) and the resulting circular-linear dichroism ratio, $C / L=\sigma_{\text {circ }}^{(2)} / \sigma_{\text {lin }}^{(2)}$. Calculated TPA tensors have been converted into the $\sigma^{(2)}$ cross-sections tabulated here using McLain's formulation ${ }^{[56,57]}$.

\begin{tabular}{|c|c|c|c|c|c|c|c|c|c|c|c|c|c|}
\hline \multicolumn{2}{|c|}{ METHOD } & \multicolumn{3}{|c|}{ CAM-B3LYP a } & \multicolumn{3}{|c|}{$\operatorname{ADC}(2)^{b}$} & \multicolumn{3}{|c|}{ EOM-CCSD } & \multicolumn{3}{|c|}{$\operatorname{CCSD}^{\mathrm{c}}$} \\
\hline & BASIS & \multicolumn{3}{|c|}{ d-Aug-cc-pVDZ } & \multicolumn{3}{|c|}{ d-Aug-cc-pVDZ } & \multicolumn{3}{|c|}{$c c-p V D Z+R$} & \multicolumn{3}{|c|}{ Ryd-TZ } \\
\hline \multicolumn{2}{|c|}{ STATE } & $\sigma(\operatorname{lin})$ & $\sigma$ (circ) & $C / L$ & $\sigma(\operatorname{lin})$ & $\sigma($ circ) & C/L & $\sigma(\operatorname{lin})$ & $\sigma($ circ) & $C / L$ & $\sigma(\operatorname{lin})$ & $\sigma($ circ) & $C / L$ \\
\hline 1 & $\pi^{*}$ & 0.032 & 0.032 & 1.01 & 0.484 & 0.465 & 0.96 & 0.06 & 0.07 & 1.10 & 0.06 & 0.02 & 0.33 \\
\hline 2 & $3 \mathrm{~s}$ & 61.17 & 90.54 & 1.48 & 116.31 & 172.76 & 1.49 & 77.44 & 114.51 & 1.48 & 94.92 & 141.31 & 1.49 \\
\hline 3 & $3 p_{z}\left(a_{1}\right)$ & 14.15 & 20.89 & 1.48 & 9.37 & 13.98 & 1.49 & 13.75 & 20.55 & 1.50 & 20.57 & 30.27 & 1.47 \\
\hline 4 & $3 p_{y}\left(b_{2}\right)$ & 143.04 & 57.09 & 0.40 & 328.60 & 81.03 & 0.25 & 107.60 & 65.85 & 0.61 & 131.87 & 55.12 & 0.42 \\
\hline 5 & $3 p_{x}\left(b_{1}\right)$ & 58.57 & 20.95 & 0.36 & 87.74 & 26.49 & 0.30 & 174.11 & 91.93 & 0.53 & 253.58 & 68.81 & 0.27 \\
\hline 6 & $3 d_{(x 2-y 2)}$ & 49.44 & 65.87 & 1.33 & 57.39 & 79.41 & 1.38 & 52.14 & 68.56 & 1.31 & & & \\
\hline 7 & $3 d$ & 234.74 & 32.97 & 0.14 & & & & 81.93 & 65.58 & 0.80 & & & \\
\hline 8 & $3 d$ & 38.33 & 40.97 & 1.07 & & & & & & & & & \\
\hline 9 & $3 d$ & 29.01 & 16.66 & 0.57 & & & & & & & & & \\
\hline 10 & $3 d$ & 25.01 & 22.37 & 0.89 & & & & & & & & & \\
\hline
\end{tabular}

${ }^{a}$ TDDFT Quadratic response theory, single residue calculations in Dalton 2018.0

${ }^{\mathrm{b}} \mathrm{ADC}(2)$ calculations using direct matrix inversion (ISR) implemented in Q-Chem 5.0

${ }^{\mathrm{c}}$ Taken from Goetz et al ${ }^{[17]}$ 


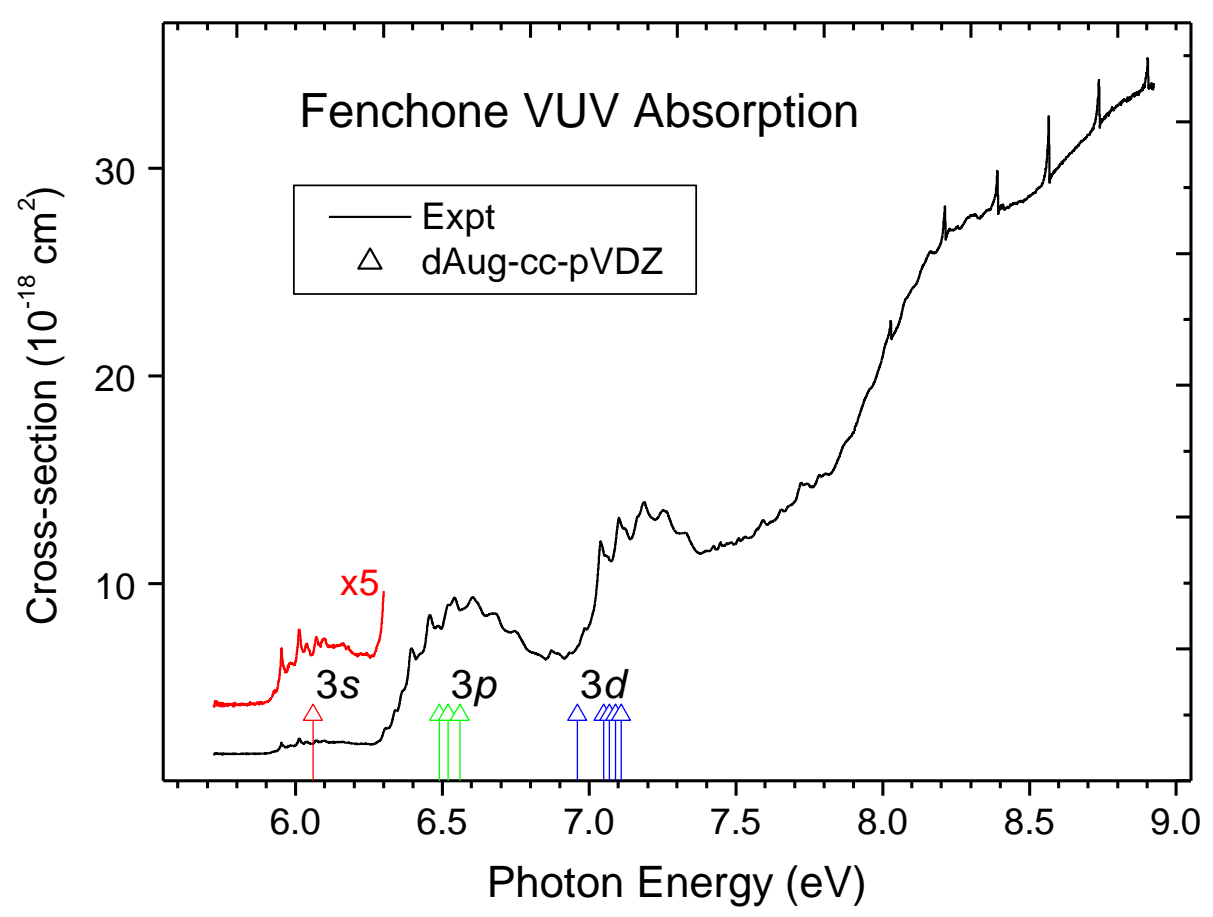

Figure 1

FT VUV absorption spectrum of room temperature fenchone vapour. The interferogram has been processed with a spectral resolution of $17.5 \mathrm{~cm}^{-1}$. Also marked are calculated vertical excitation energies of the Rydberg states, plotted with $-0.2 \mathrm{eV}$ offset to align with the experiment. 


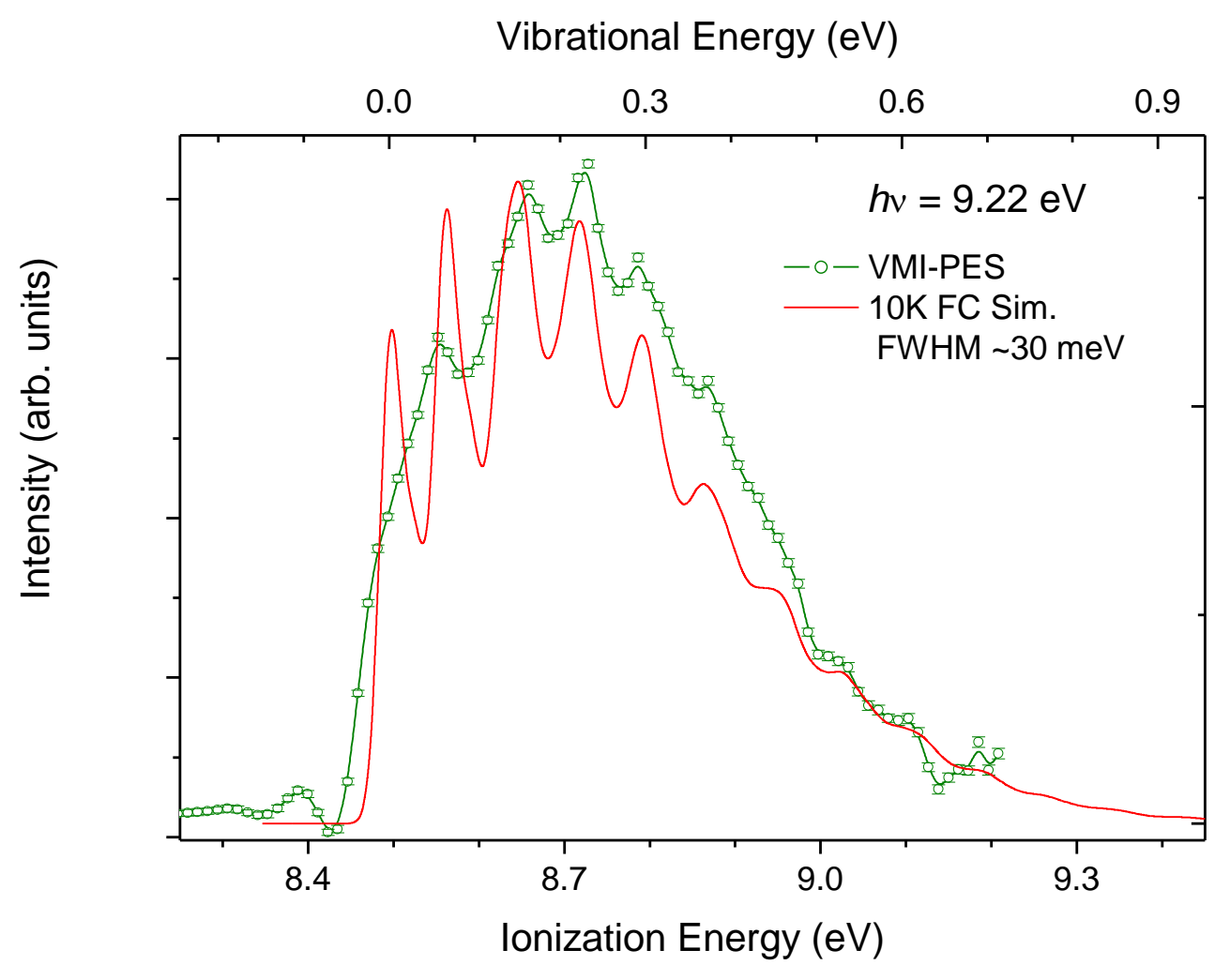

Figure 2.

Photoelectron spectrum of jet-cooled fenchone, recoded in coincidence with translationally cold parent ions ( $\mathrm{m} / \mathrm{z} 152$ ) to eliminate any contribution by dimer ionization. Also shown for comparison is a 10K Franck-Condon vibrational simulation using B3LYP/cc-pVTZ calculated harmonic frequencies. The stick spectrum has been convolved with a $230 \mathrm{~cm}^{-1} \mathrm{FWHM}$ gaussian shaping function. The features of the two spectra are empirically aligned in the plot by varying the offset between the respective energy axes, allowing the adiabatic ionization energy to be estimated as $8.495 \mathrm{eV}$. 


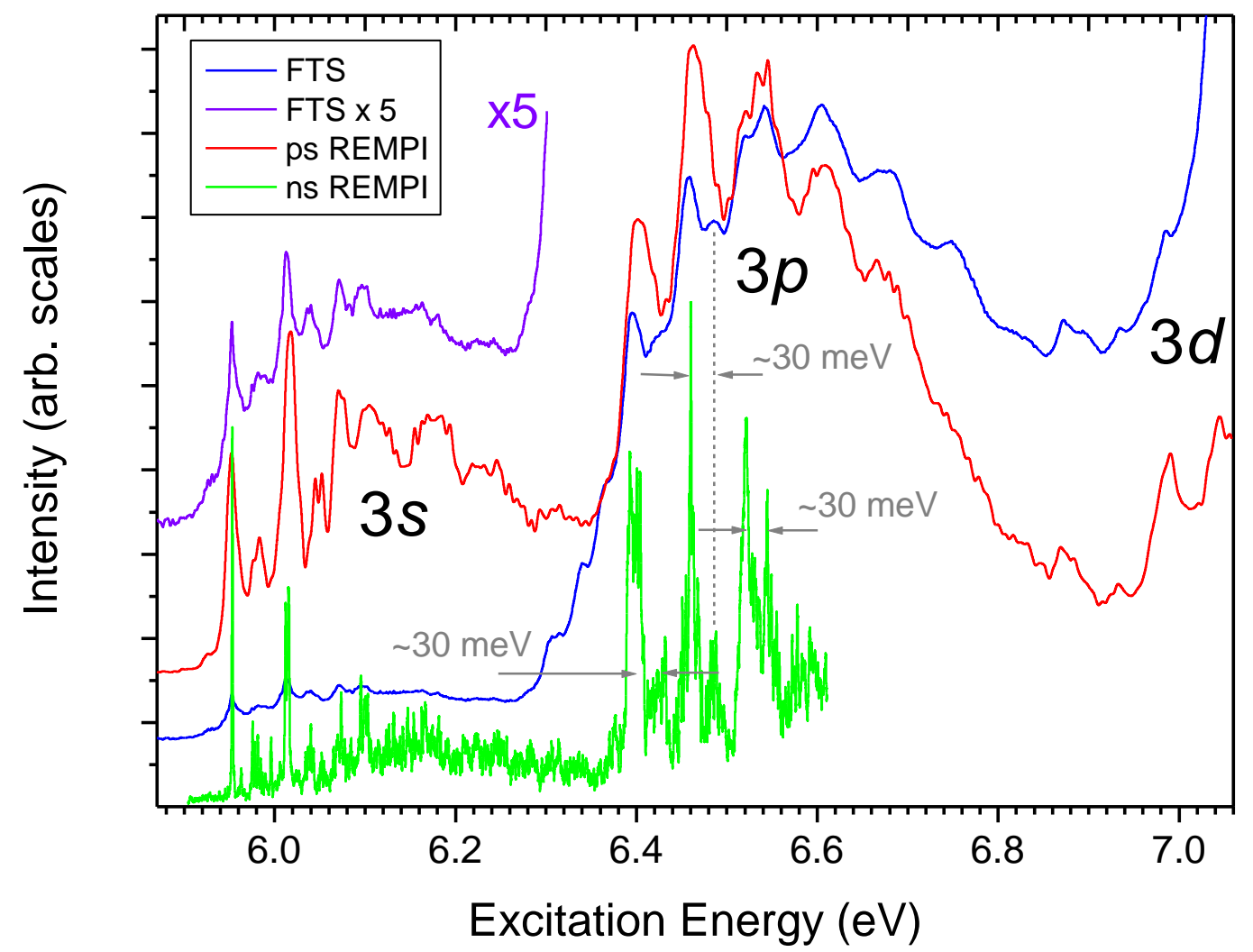

Figure 3.

ps laser REMPI spectrum of fenchone, compared with the 3spd region of the FT spectrum, and a ns laser REMPI spectrum ${ }^{[16]}$. For the REMPI spectra the plotted excitation energies are the two-photon equivalent energy. 


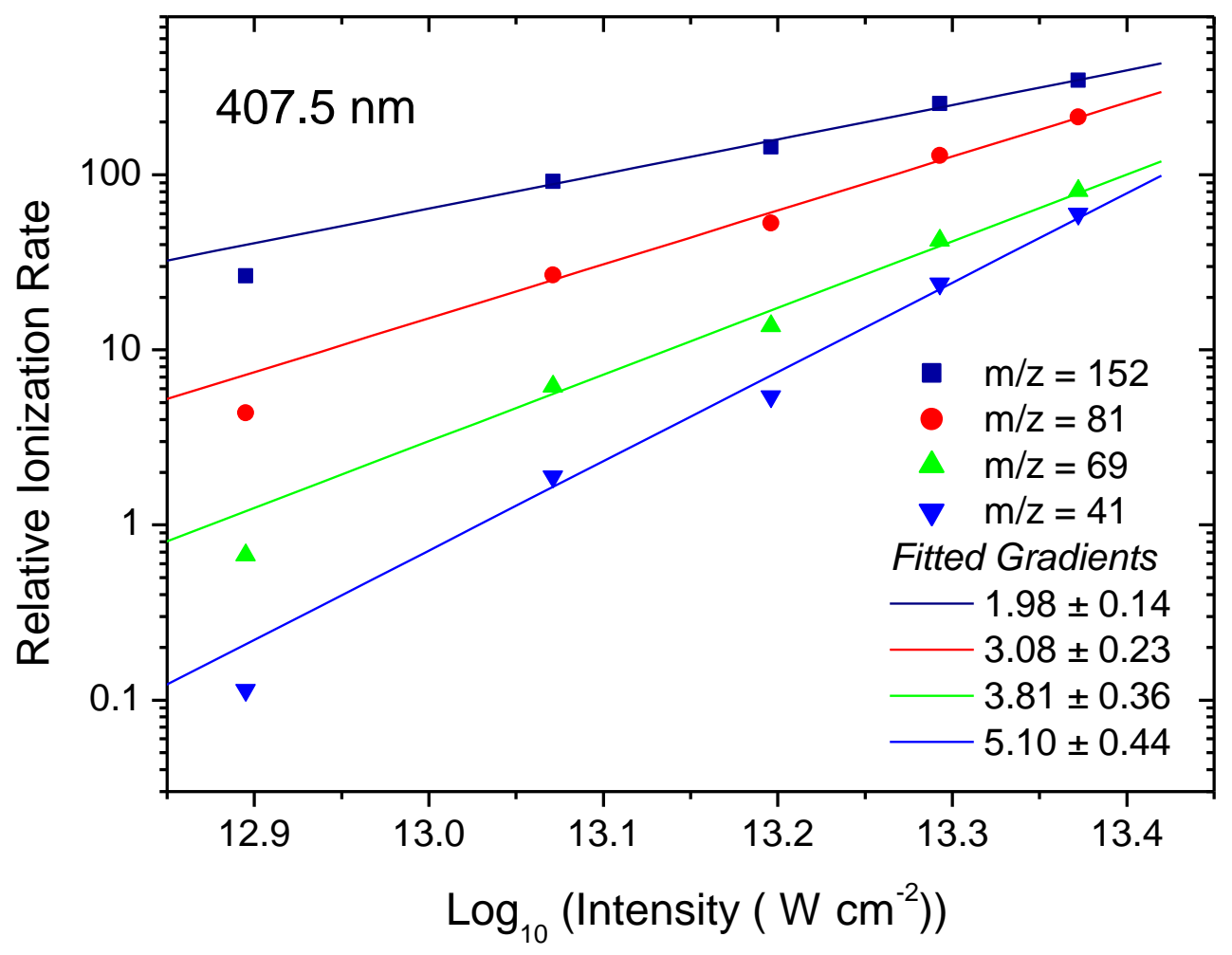

Figure 4.

Femtosecond laser intensity dependence measurements for the parent- and principal fragment-ion production ( $\lambda=407.5 \mathrm{~nm}$, linear polarization). The measurements at the lowest laser power shown (100 $\mathrm{mW}$ or $7.9 \times 10^{12} \mathrm{~W} \mathrm{~cm}^{-2}$ ) have been excluded from the linear least squares fitting performed to determine the gradients of these $\log _{10}-\log _{10}$ plots. These slopes, indicated in the key, are taken to be indicative of the $n$-photon dependence of the rate determining multiphoton ionization steps. 


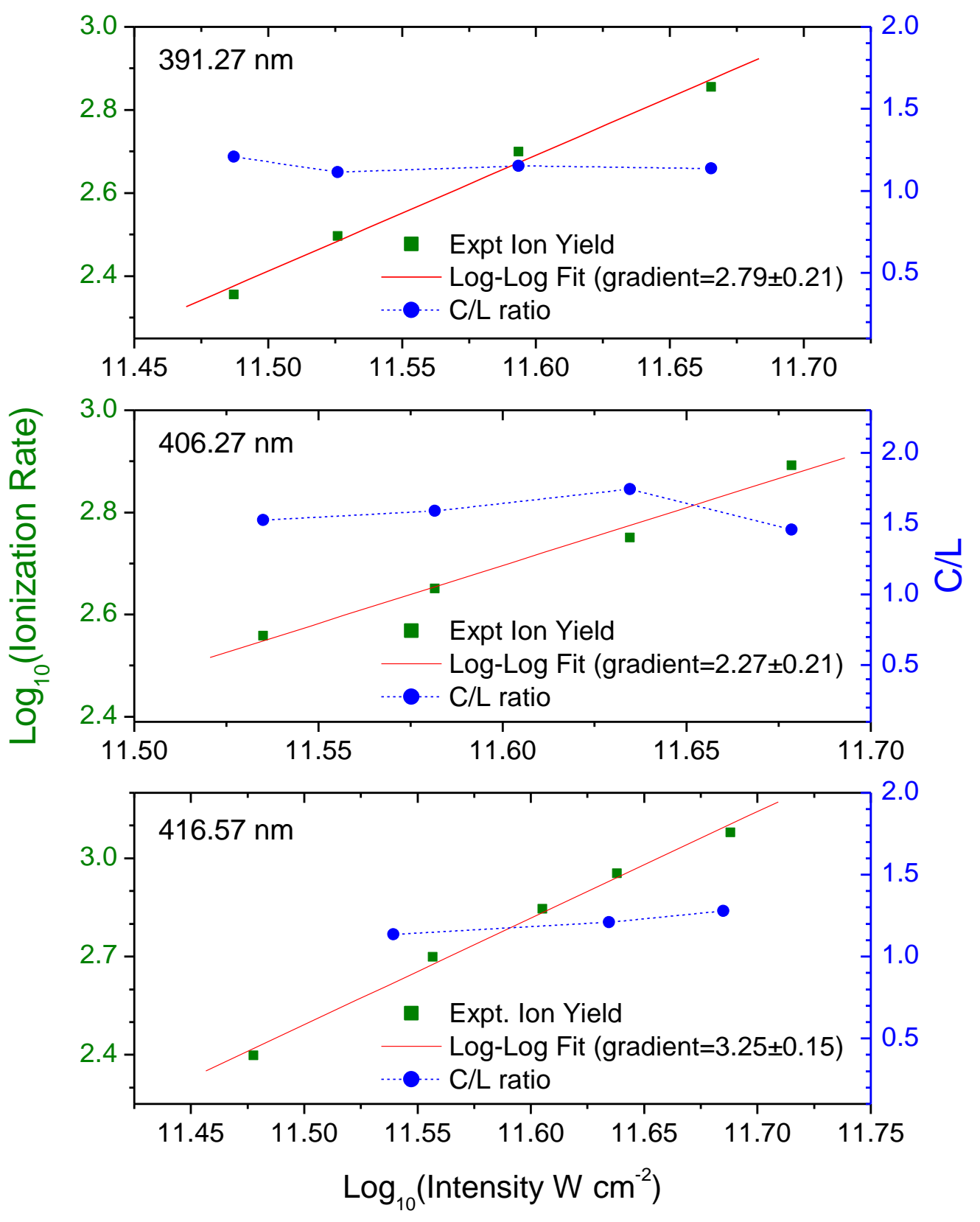

Figure 5.

Examples of the picosecond laser intensity measurements of $\mathrm{m} / \mathrm{z}=152$ ionization rates. The wavelengths chosen for presentation $(\lambda=391,406,417 \mathrm{~nm})$ lie at the extremes and centre of the $3 \mathrm{~s}$ excitation band in the REMPI spectrum. Linear least squares best fit lines are drawn through the $\log _{10}-\log _{10}$ plotted experimental data, the slopes indicating the $n$-photon dependence. Also plotted are data for the intensity dependence of the circular-linear dichroism, expressed as the ratio of ionization rate observed with circular and linear laser polarizations, C/L. Dotted lines joining these data are drawn to guide the eye. 


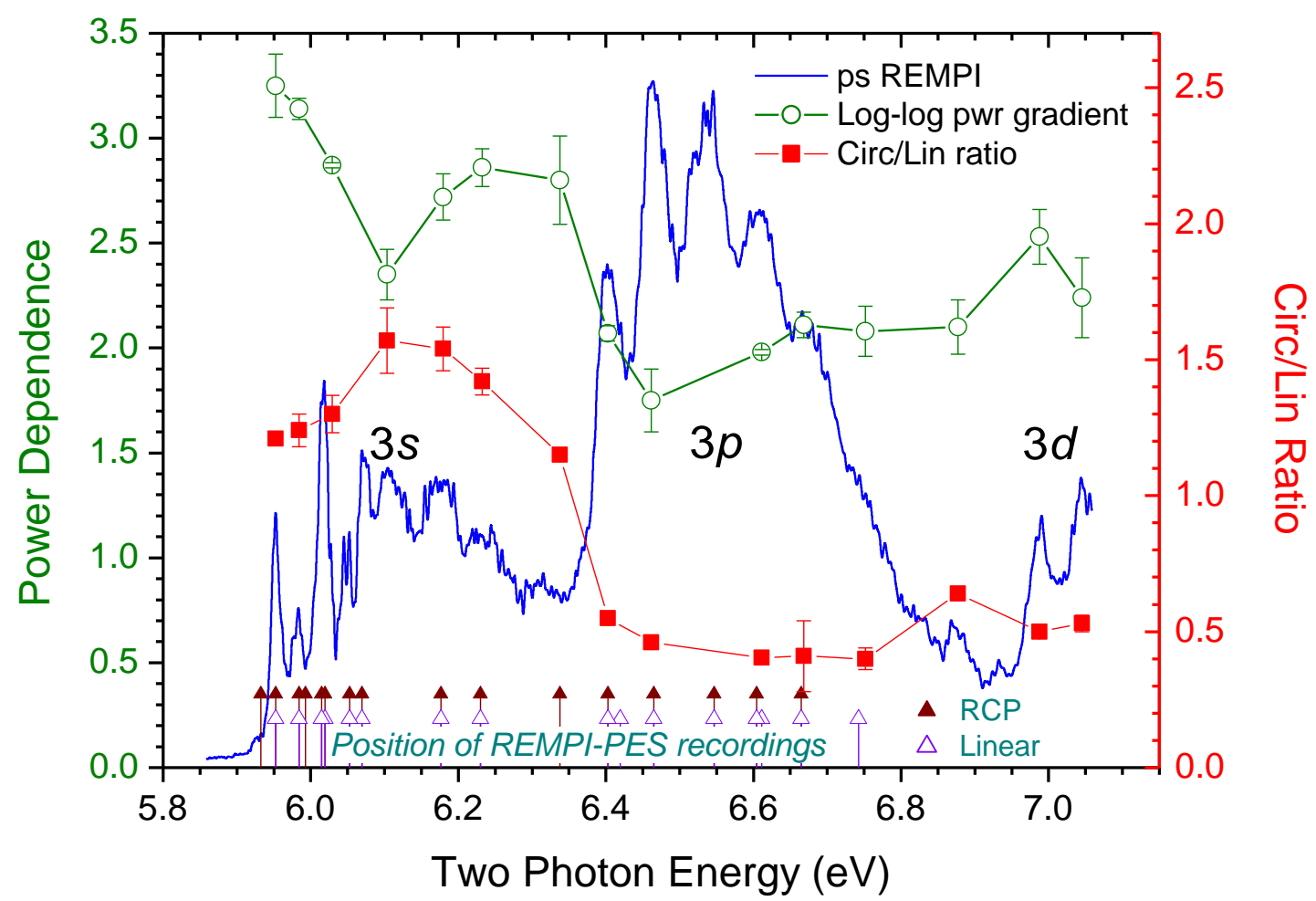

Figure 6.

Laser power dependence measurements of parent ion (m/z 152) REMPI signal, expressed as the slope of $\log _{10}-\log _{10}$ plots such as those in Fig. 5. Measurements were made with linearly polarized, picosecond duration laser pulses around a mean power $4 \times 10^{11} \mathrm{~W} \mathrm{~cm}^{-2}$. Also included are $\mathrm{C} / \mathrm{L}$ ratios of parent ion yield recorded with circular and linear polarized laser light, averaged over the 9-15 mW range. Solid lines joining data points are drawn simply to guide the eye, both for the power dependence data and the C/L ratio data. The ps REMPI spectrum is plotted on the same axis as an overall reference. Along the bottom of the figure are marked the excitation energies where REMPIPES measurements (see Fig. 7) were made, open arrow heads indicating that linear polarization was used, closed arrow heads indicating the use of RCP light. 


\section{(2+1) REMPI PES}
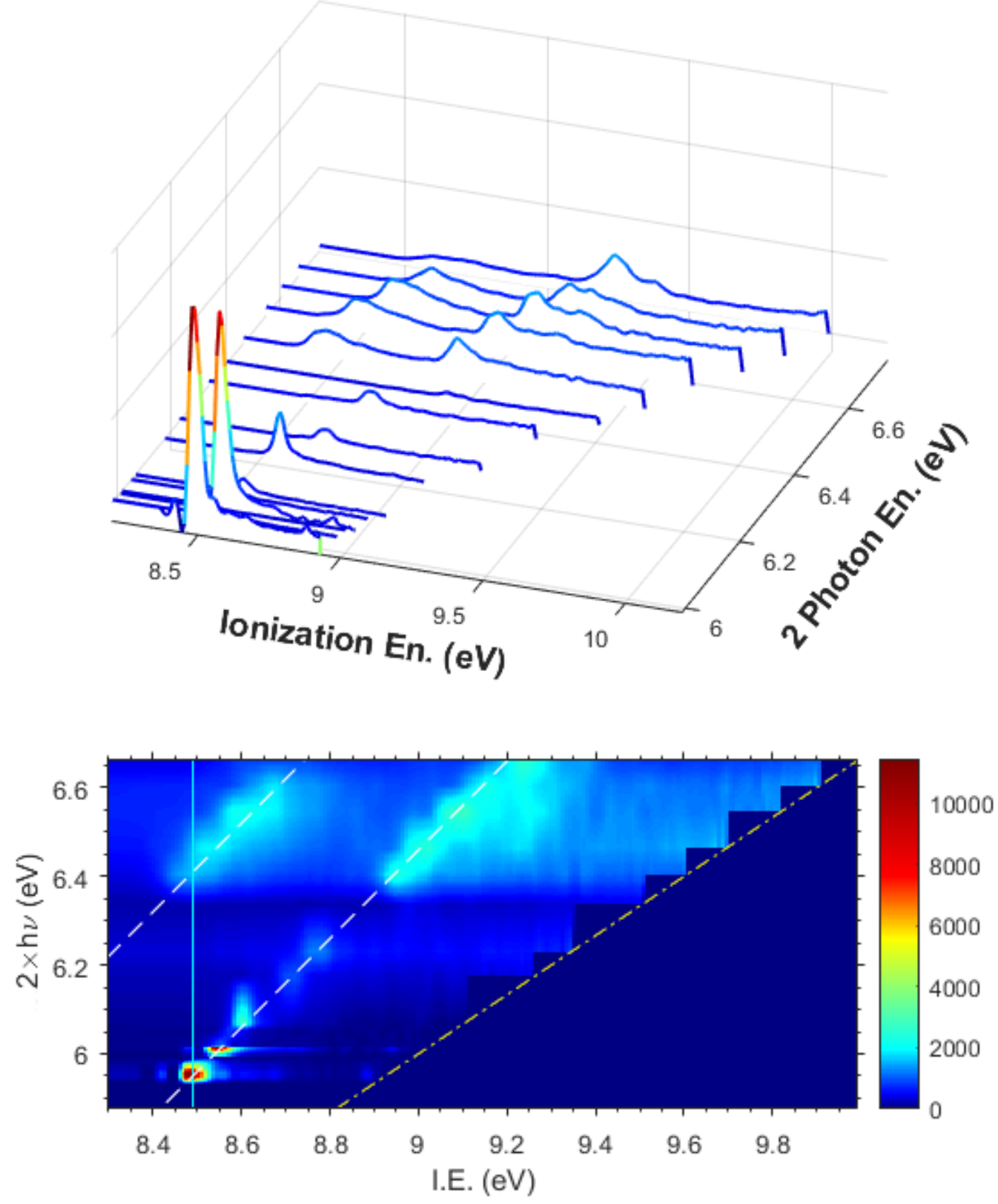

Figure 7

REMPI PES of Fenchone, recorded with picosecond duration laser pulses, for selected excitation wavelengths, shown against the two-photon equivalent energy. The ionization energy is determined by subtracting the recorded electron kinetic energy from the three-photon equivalent energy. Top panel: waterfall plot to display the spectra recorded for two-photon excitation into the $3 s p$ region. The individual circularly polarized spectra are normalized by measurement time and (laser power) ${ }^{2}$, although it is known that in the $3 \mathrm{~s}$ region the power dependence may exceed $2^{\text {nd }}$ order (see text. Lower panel: pseudo-colour intensity map of the above data. The adiabatic ionization potential 8.495 $\mathrm{eV}$ ) is marked (solid line) as is the maximum ionization energy accessible in a three-photon process at each excitation (dash-dotted line). Also marked with dashed lines are the ridges seen in the waterfall plot. 

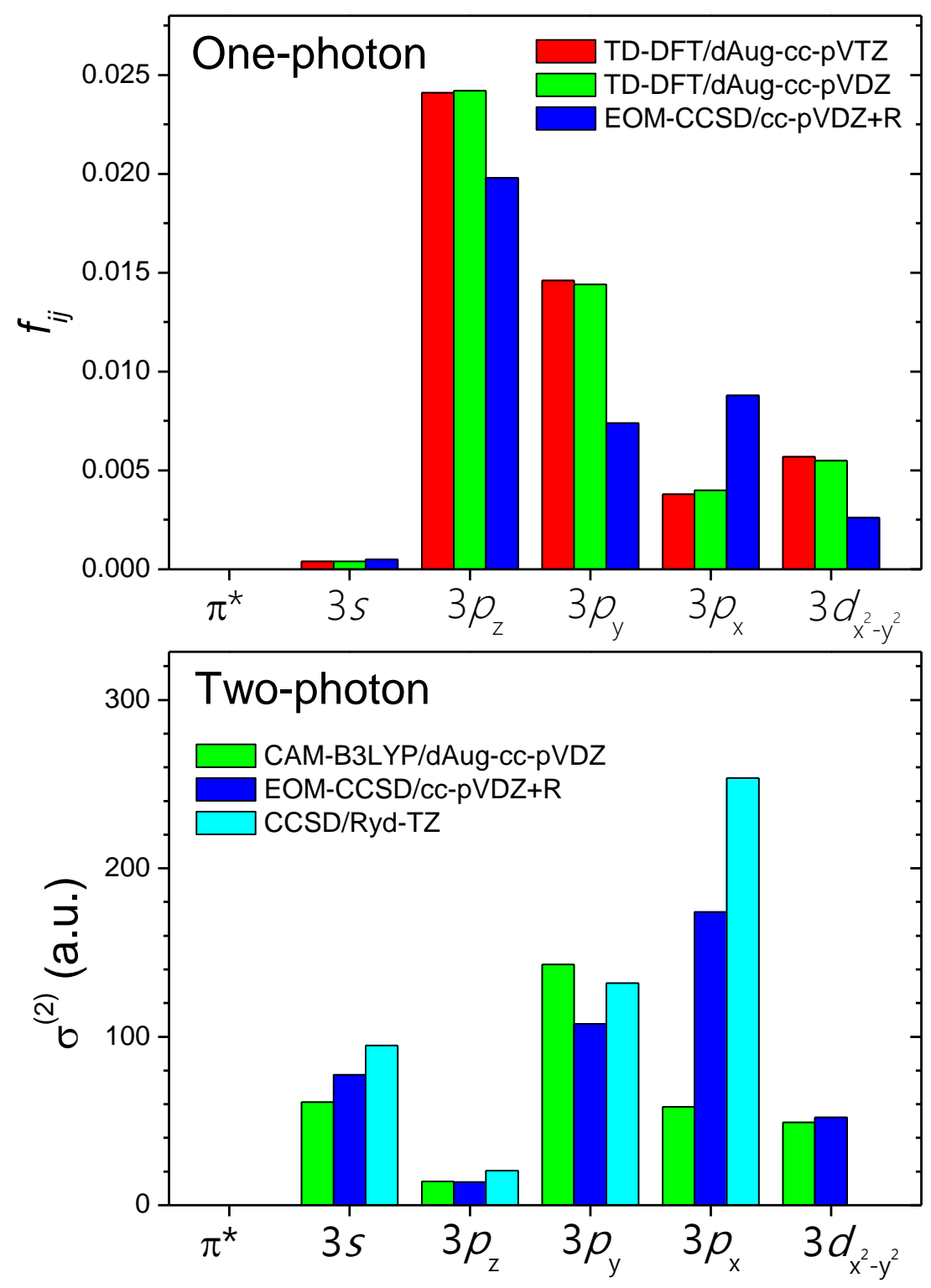

Figure 8

Comparison of calculated excitation strengths in fenchone. (top panel) oscillator strengths, $f_{i j} \propto \int \sigma^{(1)}(v) d v$, for one-photon excitation from the ground state (Table 1) ; (bottom panel) twophoton absorption cross-sections, $\sigma^{(2)}$ (atomic units). The CCSD/Ryd-TZ results are taken from Ref. [17]. 


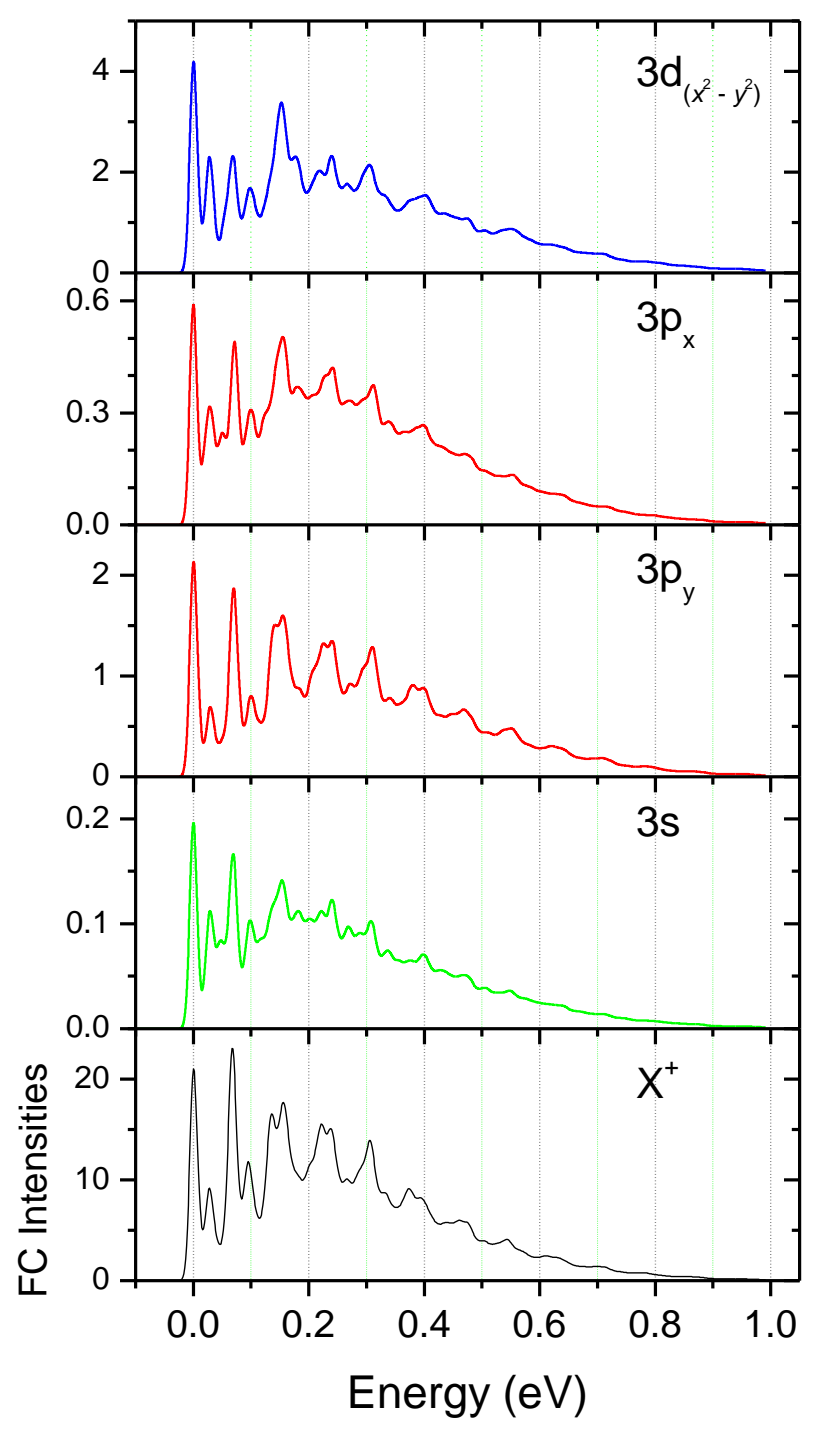

Figure 9

Franck-Condon simulations using unscaled CAM-B3LYP/SNST harmonic vibrational analysis for the cation, $X^{+}$, and several low Rydberg states of fenchone. The simulations are for cold $(10 \mathrm{~K})$ molecules and the stick spectra have been convolved with a $120 \mathrm{~cm}^{-1}$ (15 meV) FWHM gaussian for a realistic appearance. 


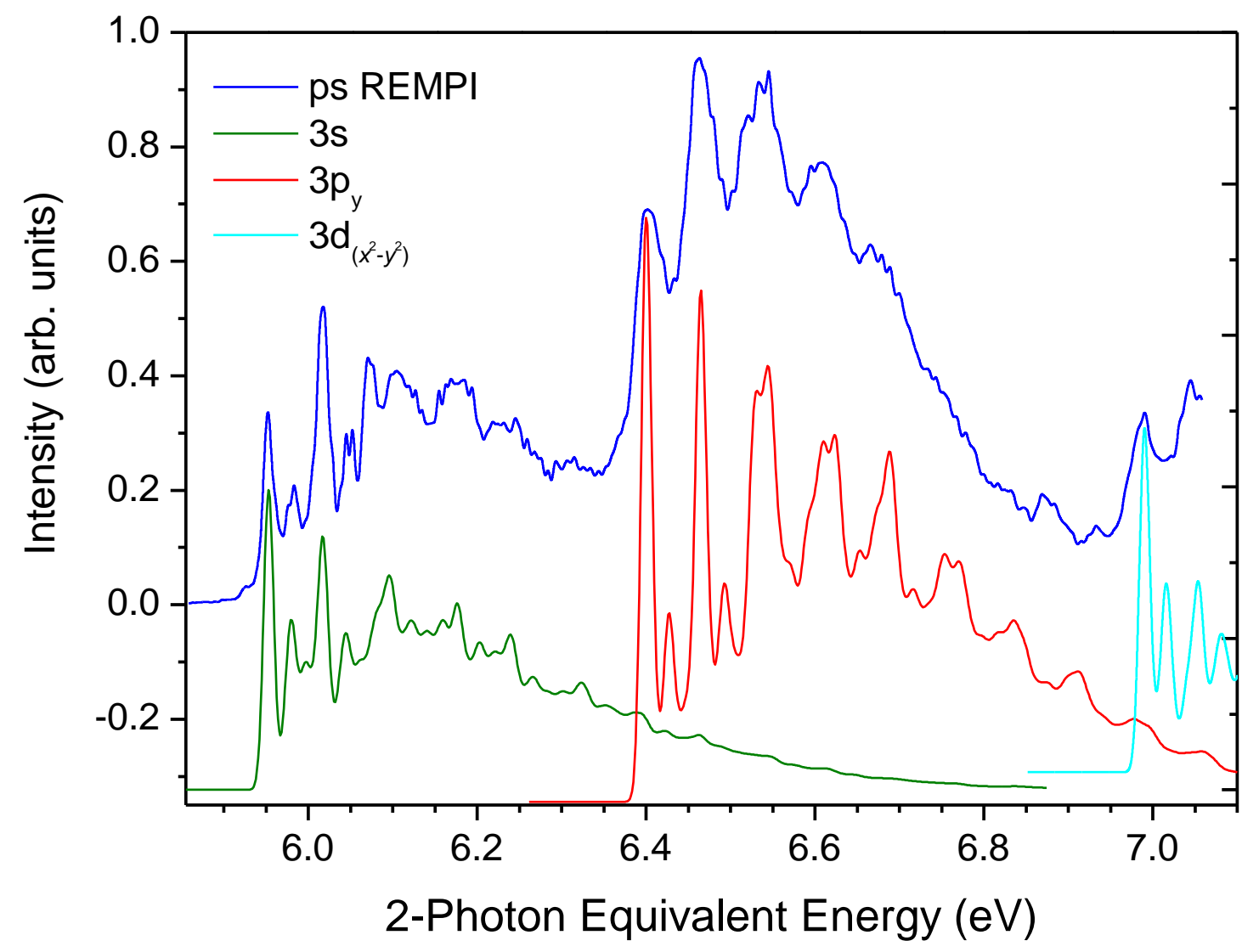

Figure 10.

Comparison of Franck-Condon vibrational simulations with the ps REMPI spectrum. The simulations are as shown in Fig. 8 except that the calculated harmonic frequencies have been scaled by a factor of 0.93 for comparison with experiment ${ }^{[68]}$. Intensities for each simulation and experiment are arbitrarily normalised for plotting. The origin of the $3 \mathrm{~s}$ simulation has been set at $5.953 \mathrm{eV}$ (to match literature values ${ }^{[16,30]}$ ) and origins of $6.40 \mathrm{eV}$ and $6.99 \mathrm{eV}$ were selected for the $3 p_{\mathrm{y}}$ and $3 d_{(\times 2-y 2)}$ simulations respectively (to best align to the experimental peak positions). 


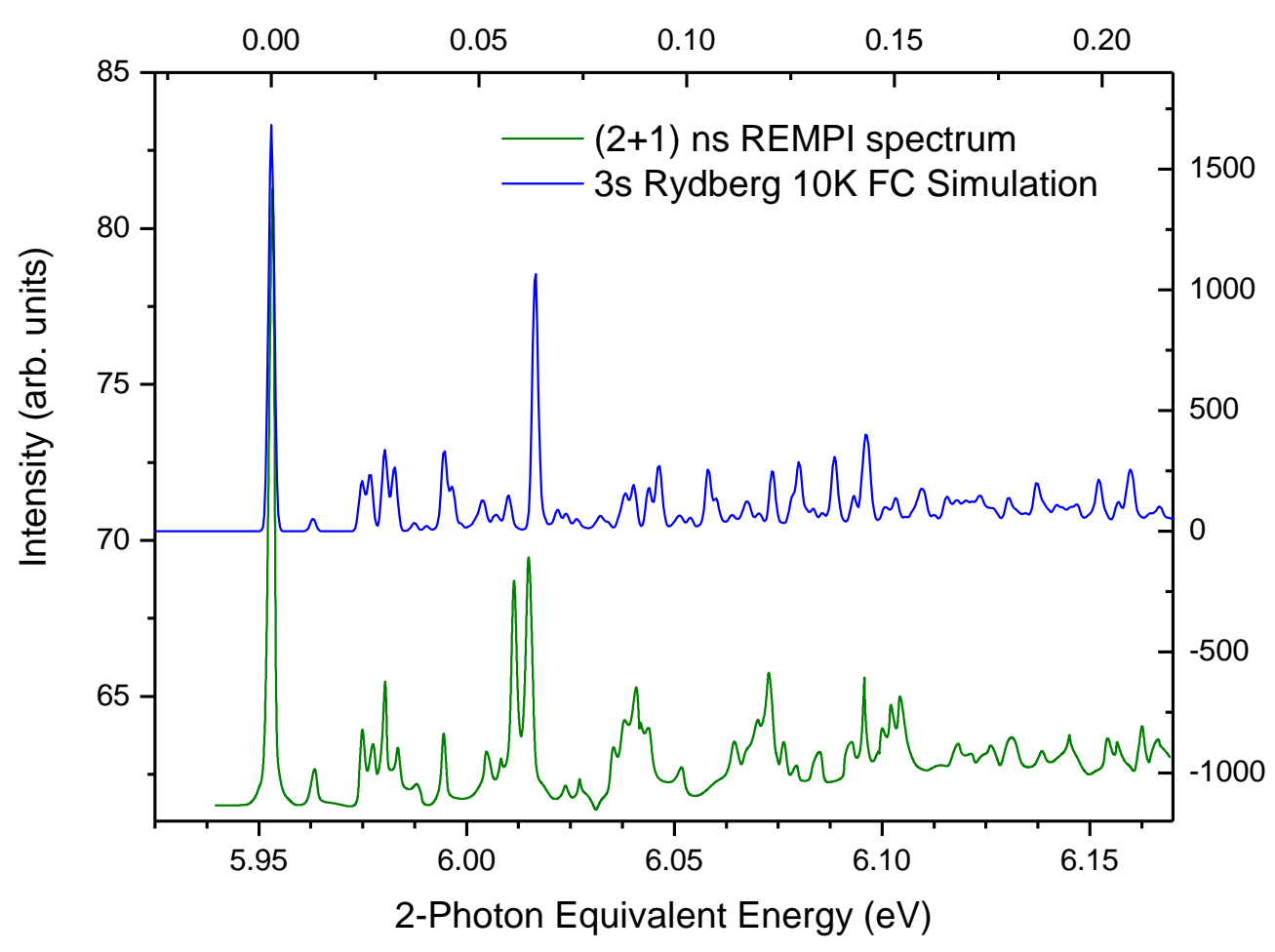

Figure 11

The $3 s$ 10K Franck-Condon simulation appearing in Figure 10, but using a reduced width gaussian function (15 $\mathrm{cm}^{-1} \mathrm{FWHM)}$ to match the higher experimental resolution of a $(2+1)$ REMPI spectrum of the fenchone $3 s$ origin region recorded by nanosecond laser excitation (taken from Ref [29]). 


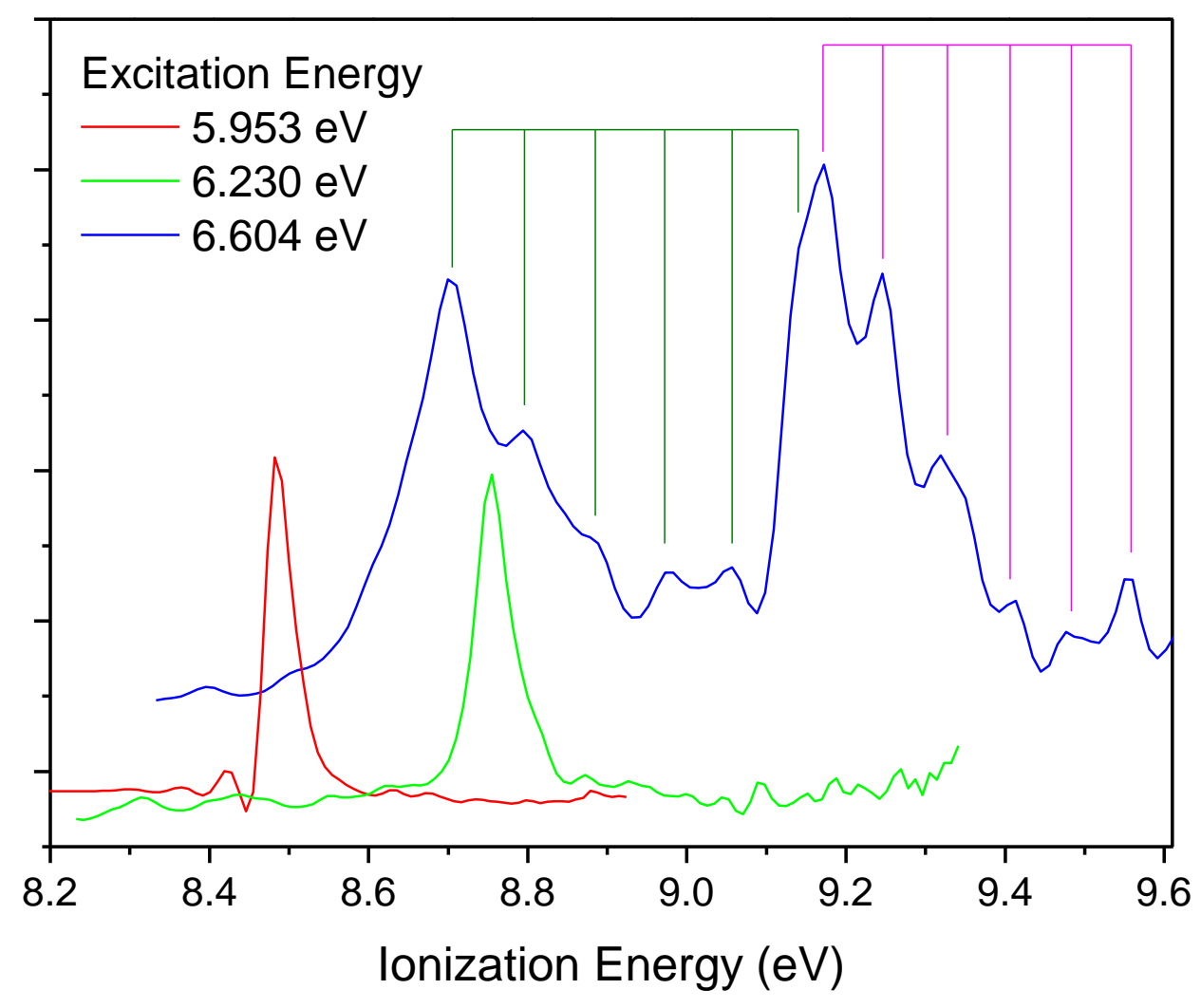

Figure 12.

Examples of the REMPI-PES, recorded using linear polarization at two-photon equivalent energies in the $3 \mathrm{~s}$ region $(5.95 \mathrm{eV}, 6.23 \mathrm{eV})$ and $3 p$ region $(6.60 \mathrm{eV})$. The intensity of each spectrum is arbitrarily normalised for clarity in the figure. 


\section{References}

[1] J. Fidler, P. M. Rodger, A. Rodger J. Chem. Soc. Perkin Trans. 2. 1993, 235-241.

[2] F. Pulm, J. Schramm, J. Hormes, S. Grimme, S. D. Peyerimhoff Chem. Phys. 1997, 224, 143-155.

[3] G. Longhi, S. Abbate, R. Gangemi, E. Giorgio, C. Rosini J. Phys. Chem. A. 2006, 110, 4958-4968.

[4] G. S. Yu, T. B. Freedman, L. A. Nafie Journal of Raman Spectroscopy. 1995, 26, 733-743.

[5] K. B. Wiberg, Y. G. Wang, M. J. Murphy, P. H. Vaccaro J. Phys. Chem. A. 2004, 108, $5559-5563$.

[6] F. J. Devlin, P. J. Stephens, J. R. Cheeseman, M. J. Frisch J. Phys. Chem. A. 1997, 101, 6322-6333.

[7] J. Fidler, P. M. Rodger, A. Rodger J. Am. Chem. Soc. 1994, 116, 7266-7273.

[8] M. Pecul, D. Marchesan, K. Ruud, S. Coriani J. Chem. Phys. 2005, 122, 024106/024101024106/024109.

[9] F. Pulm, J. Schramm, H. Lagier, J. Hormes Enantiomer. 1998, 3, 315-322.

[10] T. Muller, K. B. Wiberg, P. H. Vaccaro J. Phys. Chem. A. 2000, 104, 5959-5968.

[11] V. Ulrich, S. Barth, S. Joshi, U. Hergenhahn, E. A. Mikajlo, C. J. Harding, I. Powis J. Phys. Chem. A. 2008, 112, 3544-3549.

[12] I. Powis, C. J. Harding, G. A. Garcia, L. Nahon ChemPhysChem. 2008, 9, 475-483.

[13] L. Nahon, L. Nag, G. A. Garcia, I. Myrgorodska, U. Meierhenrich, S. Beaulieu, V. Wanie, V. Blanchet, R. Géneaux, I. Powis Phys. Chem. Chem. Phys. 2016, 18, 12696-12706.

[14] C. Lux, M. Wollenhaupt, T. Bolze, Q. Q. Liang, J. Kohler, C. Sarpe, T. Baumert Angew. Chem.-Int. Edit. 2012, 51, 5001-5005.

[15] C. Lux, M. Wollenhaupt, C. Sarpe, T. Baumert ChemPhysChem. 2015, 16, 115-137.

[16] A. Kastner, T. Ring, B. C. Kruger, G. B. Park, T. Schafer, A. Senftleben, T. Baumert J. Chem. Phys. 2017, 147, 013926.

[17] R. E. Goetz, T. A. Isaev, B. Nikoobakht, R. Berger, C. P. Koch J. Chem. Phys. 2017, 146, 024306.

[18] A. Kastner, T. Ring, H. Braun, A. Senftleben, T. Baumert ChemPhysChem. 2019, 20, 1416-1419.

[19] A. Kastner, G. Koumarianou, P. Glodic, P. C. Samartzis, N. Ladda, S. T. Ranecky, T. Ring, S. Vasudevan, C. Witte, H. Braun, H.-G. Lee, A. Senftleben, R. Berger, G. B. Park, T. Schäfer, T. Baumert Phys. Chem. Chem. Phys. 2020, 22, 7404-7411.

[20] S. Beaulieu, A. Ferre, R. Geneaux, R. Canonge, D. Descamps, B. Fabre, N. Fedorov, F. Legare, S. Petit, T. Ruchon, V. Blanchet, Y. Mairesse, B. Pons New J. Phys. 2016, 18, 102002. [21] S. Beaulieu, A. Comby, B. Fabre, D. Descamps, A. Ferré, G. Garcia, R. Géneaux, F. Légaré, L. Nahon, S. Petit, T. Ruchon, B. Pons, V. Blanchet, Y. Mairesse Faraday Discuss. 2016, 194, 325-348.

[22] A. Comby, S. Beaulieu, M. Boggio-Pasqua, D. Descamps, F. Legare, L. Nahon, S. Petit, B. Pons, B. Fabre, Y. Mairesse, V. Blanchett J. Phys. Chem. Lett. 2016, 7, 4514-4519.

[23] C. Loge, U. Boesl ChemPhysChem. 2011, 12, 1940-1947.

[24] A. Kastner, C. Lux, T. Ring, S. Zullighoven, C. Sarpe, A. Senftleben, T. Baumert ChemPhysChem. 2016, 17, 1119-1122.

[25] J. Miles, D. Fernandes, A. Young, C. M. M. Bond, S. W. Crane, O. Ghafur, D. Townsend, J. Sá, J. B. Greenwood Analytica Chimica Acta. 2017, 984, 134-139.

[26] D. C. Frost, N. P. C. Westwood, N. H. Werstiuk Can. J. Chem.-Rev. Can. Chim. 1980, 58, $1659-1665$.

[27] J. Pollmann, R. Franke, J. Hormes Spectrochimica Acta Part A-Molecular and Biomolecular Spectroscopy. 1997, 53, 491-493. 
[28] M. Getzlaff, G. Schönhense J. Elec. Spec. Rel. Phen. 1998, 95, 225-230.

[29] J. W. Driscoll, T. Baer, T. J. Cornish J. Mol. Struct. 1991, 249, 95-107.

[30] T. J. Cornish, T. Baer J. Am. Chem. Soc. 1988, 110, 6287-6291.

[31] N. de Oliveira, M. Roudjane, D. Joyeux, D. Phalippou, J.-C. Rodier, L. Nahon Nature Photonics. 2011, 5, 149-153.

[32] N. de Oliveira, D. Joyeux, M. Roudjane, J.-F. Gil, B. Pilette, L. Archer, K. Ito, L. Nahon J. Synchrot. Radiat. 2016, 23, 887-900.

[33] L. Nahon, N. d. Oliveira, G. Garcia, J. F. Gil, B. Pilette, O. Marcouille, B. Lagarde, F. Polack J. Synchrot. Radiat. 2012, 19, 508-520.

[34] M. A. Smialek, M. J. Hubin-Franskin, J. Delwiche, D. Duflot, N. J. Mason, S. VronningHoffmann, G. G. B. de Souza, A. M. F. Rodrigues, F. N. Rodrigues, P. Limao-Vieira Phys. Chem. Chem. Phys. 2012, 14, 2056-2064.

[35] G. A. Garcia, B. K. C. de Miranda, M. Tia, S. Daly, L. Nahon Rev. Sci. Inst. 2013, 84, 053112 .

[36] S. Daly, I. Powis, G. A. Garcia, M. Tia, L. Nahon J. Chem. Phys. 2017, 147, 013937.

[37] S. M. Bellm, K. L. Reid Chem. Phys. Lett. 2004, 395, 253-258.

[38] G. A. Garcia, L. Nahon, I. Powis Rev. Sci. Inst. 2004, 75, 4989-4996.

[39] Z. H. Qu, Z. B. Qin, X. F. Zheng, H. Wang, G. X. Yao, X. Y. Zhang, Z. F. Cui Spectrochimica Acta Part A-Molecular and Biomolecular Spectroscopy. 2017, 173, 432-438.

[40] D. Irimia, D. Dobrikov, R. Kortekaas, H. Voet, D. A. van den Ende, W. A. Groen, M. H. M. Janssen Rev. Sci. Inst. 2009, 80, 113303.

[41] A. Vredenborg, W. G. Roeterdink, M. H. M. Janssen Rev. Sci. Inst. 2008, 79, 063108.

[42] M. M. Rafiee Fanood, I. Powis, M. H. M. Janssen J. Phys. Chem. A. 2014, 118, 1154111546.

[43] M. M. Rafiee Fanood, M. H. M. Janssen, I. Powis J. Chem. Phys. 2016, 145, 124320.

[44] M. J. Frisch, G. W. Trucks, H. B. Schlegel, G. E. Scuseria, M. A. Robb, J. R. Cheeseman, G. Scalmani, V. Barone, G. A. Petersson, H. Nakatsuji, X. Li, M. Caricato, A. V. Marenich, J. Bloino, B. G. Janesko, R. Gomperts, B. Mennucci, H. P. Hratchian, J. V. Ortiz, A. F. Izmaylov, J. L. Sonnenberg, D. Williams-Young, F. Ding, F. Lipparini, F. Egidi, J. Goings, B. Peng, A. Petrone, T. Henderson, D. Ranasinghe, V. G. Zakrzewski, J. Gao, N. Rega, G. Zheng, W. Liang, M. Hada, M. Ehara, K. Toyota, R. Fukuda, J. Hasegawa, M. Ishida, T. Nakajima, Y. Honda, O. Kitao, H. Nakai, T. Vreven, K. Throssell, J. J. A. Montgomery, J. E. Peralta, F. Ogliaro, M. Bearpark, J. J. Heyd, E. Brothers, K. N. Kudin, V. N. Staroverov, T. A. Keith, R. Kobayashi, J. Normand, K. Raghavachari, A. Rendell, J. C. Burant, S. S. Iyengar, J. Tomasi, M. Cossi, J. M. Millam, M. Klene, C. Adamo, R. Cammi, J. W. Ochterski, R. L. Martin, K. Morokuma, O. Farkas, J. B. Foresman, D. J. Fox in Gaussian 16 Revision A.03, Vol. (Ed.^Eds.: Editor), Gaussian Inc., City, 2016.

[45] M. Hodecker, M. Biczysko, A. Dreuw, V. Barone J Chem Theory Comput. 2016, 12, 2820 2833.

[46] SNST https://smart.sns.it (accessed Jan 21 2020)

[47] M. Caricato, G. W. Trucks, M. J. Frisch, K. B. Wiberg J Chem Theory Comput. 2010, 6, 370-383.

[48] M. Caricato, G. W. Trucks, M. J. Frisch, K. B. Wiberg J Chem Theory Comput. 2011, 7, 456-466.

[49] Y. Shao, Z. Gan, E. Epifanovsky, A. T. B. Gilbert, M. Wormit, J. Kussmann, A. W. Lange, A. Behn, J. Deng, X. Feng, D. Ghosh, M. Goldey, P. R. Horn, L. D. Jacobson, I. Kaliman, R. Z. Khaliullin, T. Kuś, A. Landau, J. Liu, E. I. Proynov, Y. M. Rhee, R. M. Richard, M. A. Rohrdanz, R. P. Steele, E. J. Sundstrom, H. L. Woodcock, P. M. Zimmerman, D. Zuev, B. Albrecht, E. Alguire, B. Austin, G. J. O. Beran, Y. A. Bernard, E. Berquist, K. Brandhorst, K. B. Bravaya, S. T. Brown, D. Casanova, C.-M. Chang, Y. Chen, S. H. Chien, K. D. Closser, D. 
L. Crittenden, M. Diedenhofen, R. A. DiStasio, H. Do, A. D. Dutoi, R. G. Edgar, S. Fatehi, L. Fusti-Molnar, A. Ghysels, A. Golubeva-Zadorozhnaya, J. Gomes, M. W. D. Hanson-Heine, P. H. P. Harbach, A. W. Hauser, E. G. Hohenstein, Z. C. Holden, T.-C. Jagau, H. Ji, B. Kaduk, K. Khistyaev, J. Kim, J. Kim, R. A. King, P. Klunzinger, D. Kosenkov, T. Kowalczyk, C. M. Krauter, K. U. Lao, A. D. Laurent, K. V. Lawler, S. V. Levchenko, C. Y. Lin, F. Liu, E. Livshits, R. C. Lochan, A. Luenser, P. Manohar, S. F. Manzer, S.-P. Mao, N. Mardirossian, A. V. Marenich, S. A. Maurer, N. J. Mayhall, E. Neuscamman, C. M. Oana, R. Olivares-Amaya, D. P. O’Neill, J. A. Parkhill, T. M. Perrine, R. Peverati, A. Prociuk, D. R. Rehn, E. Rosta, N. J. Russ, S. M. Sharada, S. Sharma, D. W. Small, A. Sodt, T. Stein, D. Stück, Y.-C. Su, A. J. W. Thom, T. Tsuchimochi, V. Vanovschi, L. Vogt, O. Vydrov, T. Wang, M. A. Watson, J. Wenzel, A. White, C. F. Williams, J. Yang, S. Yeganeh, S. R. Yost, Z.-Q. You, I. Y. Zhang, X. Zhang, Y. Zhao, B. R. Brooks, G. K. L. Chan, D. M. Chipman, C. J. Cramer, W. A. Goddard, M. S. Gordon, W. J. Hehre, A. Klamt, H. F. Schaefer, M. W. Schmidt, C. D. Sherrill, D. G. Truhlar, A. Warshel, X. Xu, A. Aspuru-Guzik, R. Baer, A. T. Bell, N. A. Besley, J.-D. Chai, A. Dreuw, B. D. Dunietz, T. R. Furlani, S. R. Gwaltney, C.-P. Hsu, Y. Jung, J. Kong, D. S. Lambrecht, W. Liang, C. Ochsenfeld, V. A. Rassolov, L. V. Slipchenko, J. E. Subotnik, T. Van Voorhis, J. M. Herbert, A. I. Krylov, P. M. W. Gill, M. Head-Gordon Mol. Phys. 2015, 113, 184-215.

[50] K. Kaufmann, W. Baumeister, M. Jungen J. Phys. B-At. Mol. Opt. Phys. 1989, 22, 2223 2240.

[51] H. Ganjitabar, D. P. Singh, R. Chapman, A. Gardner, R. S. Minns, I. Powis, K. L. Reid, A. Vredenborg Mol. Phys. 2020, e1808907.

[52] V. Rishi, A. Perera, M. Nooijen, R. J. Bartlett The Journal of Chemical Physics. 2017, $146,144104$.

[53] J. J. Goings, M. Caricato, M. J. Frisch, X. Li The Journal of Chemical Physics. 2014, 141, 164116.

[54] K. Aidas, C. Angeli, K. L. Bak, V. Bakken, R. Bast, L. Boman, O. Christiansen, R. Cimiraglia, S. Coriani, P. Dahle, E. K. Dalskov, U. Ekström, T. Enevoldsen, J. J. Eriksen, P. Ettenhuber, B. Fernández, L. Ferrighi, H. Fliegl, L. Frediani, K. Hald, A. Halkier, C. Hättig, H. Heiberg, T. Helgaker, A. C. Hennum, H. Hettema, E. Hjertenæs, S. Høst, I.-M. Høyvik, M. F. Iozzi, B. Jansík, H. J. A. Jensen, D. Jonsson, P. Jørgensen, J. Kauczor, S. Kirpekar, T. Kjærgaard, W. Klopper, S. Knecht, R. Kobayashi, H. Koch, J. Kongsted, A. Krapp, K. Kristensen, A. Ligabue, O. B. Lutnæs, J. I. Melo, K. V. Mikkelsen, R. H. Myhre, C. Neiss, C. B. Nielsen, P. Norman, J. Olsen, J. M. H. Olsen, A. Osted, M. J. Packer, F. Pawlowski, T. B. Pedersen, P. F. Provasi, S. Reine, Z. Rinkevicius, T. A. Ruden, K. Ruud, V. V. Rybkin, P. Sałek, C. C. M. Samson, A. S. de Merás, T. Saue, S. P. A. Sauer, B. Schimmelpfennig, K. Sneskov, A. H. Steindal, K. O. Sylvester-Hvid, P. R. Taylor, A. M. Teale, E. I. Tellgren, D. P. Tew, A. J. Thorvaldsen, L. Thøgersen, O. Vahtras, M. A. Watson, D. J. D. Wilson, M. Ziolkowski, H. Ågren WIREs Computational Molecular Science. 2014, 4, $269-284$.

[55] S. Knippenberg, D. R. Rehn, M. Wormit, J. H. Starcke, I. L. Rusakova, A. B. Trofimov, A. Dreuw J Chem Phys. 2012, 136, 064107.

[56] W. M. McClain, R. A. Harris in Two-Photon Molecular Spectroscopy in Liquids and Gases, Vol. 3 (Ed. E. C. Lim), Academic Press., New York, 1977, pp.1-56.

[57] P. R. Monson, W. M. McClain J. Chem. Phys. 1970, 53, 29-\&.

[58] F. Avila Ferrer, F. Santoro Phys. Chem. Chem. Phys. 2012, 14, 13549-13563.

[59] K. P. Gross, O. Schnepp J. Chem. Phys. 1978, 68, 2647-2657.

[60] D. Kubala, E. A. Drage, A. M. E. Al-Faydhi, J. Kocisek, P. Papp, V. Matejcik, P. Mach, J. Urban, P. Limao-Vieira, S. V. Hoffmann, S. Matejcik, N. J. Mason Int. J. Mass. Spec. 2009, 280, 169-173.

[61] J. A. Myer, J. A. R. Samson The Journal of Chemical Physics. 1970, 52, 266-271. 
[62] William E. Wallace "Mass Spectra", NIST Mass Spectrometry Data Center, in NIST Chemistry WebBook, NIST Standard Reference Database Number 69, Eds. P.J. Linstrom and W.G. Mallard, National Institute of Standards and Technology, Gaithersburg MD, 20899, 2020 [63] W. Dietz, H. J. Neusser, U. Boesl, E. W. Schlag, S. H. Lin Chem. Phys. 1982, 66, 105127.

[64] P. R. Monson, W. M. McClain J. Chem. Phys. 1972, 56, 4817-\&.

[65] J. A. Tiburcio-Moreno, J. J. Alvarado-Gil, C. Diaz, L. Echevarria, F. E. Hernandez Chem. Phys. Lett. 2013, 583, 160-164.

[66] M. G. Vivas, D. L. Silva, L. De Boni, Y. Bretonniere, C. Andraud, F. Laibe-Darbour, J. C. Mulatier, R. Zalesny, W. Bartkowiak, S. Canuto, C. R. Mendonca J. Phys. Chem. Lett. 2013, 4, 1753-1759.

[67] M. T. P. Beerepoot, D. H. Friese, N. H. List, J. Kongsted, K. Ruud Phys. Chem. Chem. Phys. 2015, 17, 19306-19314.

[68] J. P. Merrick, D. Moran, L. Radom J. Phys. Chem. A. 2007, 111, 11683-11700.

[69] T. Baer in Theory and energetics of mass spectrometry, Vol. 6 (Ed. R. A. W. Johnstone), The Royal Society of Chemistry, 1981, pp.1-58.

[70] M. Stener, P. Decleva, A. Lisini J. Elec. Spec. Rel. Phen. 1995, 74, 29-43.

[71] M. Stener, G. Fronzoni, D. Toffoli, P. Decleva Chem. Phys. 2002, 282, 337-351.

[72] M. Stener, D. D. Tommaso, G. Fronzoni, P. Decleva, I. Powis J. Chem. Phys. 2006, 124, 024326.

[73] M. G. Vivas, L. De Boni, Y. Bretonniere, C. Andraud, C. R. Mendonca Opt. Express. 2012, 20, 18600-18608.

[74] L. De Boni, C. Toro, F. E. Hernandez Opt. Lett. 2008, 33, 2958-2960.

[75] D. H. Parker, J. O. Berg, M. A. Elsayed Chem. Phys. Lett. 1978, 56, 197-199.

[76] J. O. Berg, D. H. Parker, M. A. Elsayed J. Chem. Phys. 1978, 68, 5661-5663.

[77] B. A. Heath, G. J. Fisanick, M. B. Robin, T. S. Eichelberger J. Chem. Phys. 1980, 72, 5991-5997. 


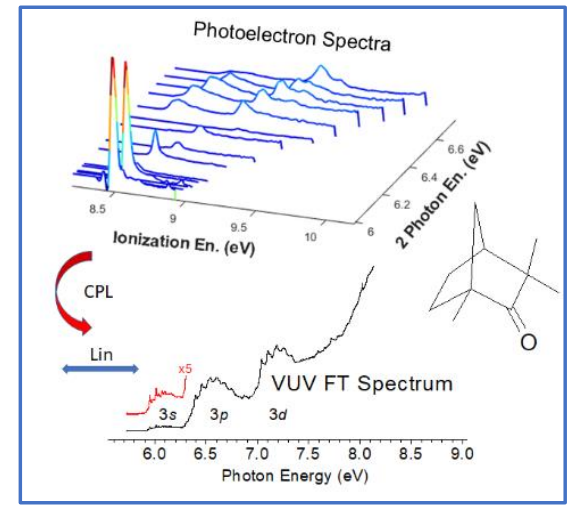

Using Fourier Transform VUV photon absorption spectroscopy and $(2+1)$ resonance-enhanced multiphoton (REMPI) spectroscopy we examine the $3 s p(d)$ Rydberg states of fenchone. Circular-linear dichroism observed in the polarized laser REMPI ionization yields is interpreted to help identify the contribution of the $3 p$ sub-states. Experimental interpretation is further aided by computational modelling of the two-photon excitation of the Rydberg intermediates. Further insight into the vibronic state-selected Rydberg photoionization and relaxation processes is acquired by mapping the vibrationally resolved REMPI photoelectron spectroscopy.

79 words 